\title{
Method to compute the stress-energy tensor for a quantized scalar field when a black hole forms from the collapse of a null shell
}

\author{
Paul R. Anderson $\oplus^{1, *}$ Shohreh Gholizadeh Siahmazgi, ${ }^{1, \dagger}$ Raymond D. Clark, ${ }^{1}$ and Alessandro Fabbri $\odot^{2,3, \#}$ \\ ${ }^{1}$ Department of Physics, Wake Forest University, Winston-Salem, North Carolina 27109, USA \\ ${ }^{2}$ Departamento de Física Teórica and IFIC, Universidad de Valencia-CSIC, \\ C. Dr. Moliner 50, 46100 Burjassot, Spain \\ ${ }^{3}$ Université Paris-Saclay, CNRS/IN2P3, IJC Lab, 91405 Orsay Cedex, France
}

(Received 14 September 2020; accepted 13 November 2020; published 31 December 2020)

\begin{abstract}
A method is given to compute the stress-energy tensor for a massless minimally coupled scalar field in a spacetime where a black hole forms from the collapse of a spherically symmetric null shell in four dimensions. Part of the method involves matching the modes for the in vacuum state to a complete set of modes in Schwarzschild spacetime. The other part involves subtracting from the unrenormalized expression for the stress-energy tensor when the field is in the in vacuum state, the corresponding expression when the field is in the Unruh state and adding to this the renormalized stress-energy tensor for the field in the Unruh state. The method is shown to work in the two-dimensional case where the results are known.
\end{abstract}

DOI: $10.1103 /$ PhysRevD.102.125035

\section{INTRODUCTION}

The stress-energy tensor of a quantized field is an extremely useful tool for studying quantum effects in curved space because it takes both particle production and vacuum polarization into account. It can be computed in a background spacetime to obtain the energy density, pressure, etc. for a quantum field in that spacetime. It can also be used in the context of semiclassical gravity to compute the backreaction of the quantum field on the spacetime geometry.

For black holes in four-dimensional, 4D, spacetimes, the full stress-energy tensor must be computed numerically. This is a difficult task that has to date only been done without other approximations for the cases of static spherically symmetric black holes [1-15] and the stationary Kerr metric $[16,17]$. However, because of the difficulty involved, to our knowledge, no one has numerically computed the full stress-energy tensor for a quantized field in a 4D spacetime in which a black hole forms from collapse. This is important because there can be a significant difference between the stress-energy tensor for a quantum field in a $2 \mathrm{D}$ versus a $4 \mathrm{D}$ spacetime such as that found for a massless minimally coupled scalar field in an extreme ReissnerNordstrom spacetime $[9,18]$.

In this paper we present a method to compute the renormalized stress-energy tensor, $\left\langle\right.$ in $\left|T_{a b}\right|$ in $\rangle$, for a massless

\footnotetext{
*anderson@wfu.edu

†hols18@wfu.edu

¥afabbri@ific.uv.es
}

minimally coupled scalar field in the case that a black hole forms from the collapse of a spherically symmetric null shell. This model has been previously used to derive the Hawking effect $[19,20]$, investigate how the stress-energy tensor is affected by the production of a pair of particles due to the Hawking effect [21], study some details of how the spectrum and number of produced particles changes in time during and after the collapse [22,23], and in 2D to compute the stress-energy tensor for a massless minimally coupled scalar field $[20,24]$. While this is not a realistic model for collapse because the shell begins with an infinite size, this is probably the simplest model to work with that involves collapse in 4D to form a black hole. Thus it is a reasonable first choice for the full numerical computation of the stress-energy tensor of a quantized field in a 4D spacetime in which a black hole forms from collapse. Further, since the Hawking effect is independent of how the black hole forms [25], and since it is expected that the stress-energy tensor at late times will also be independent of the formation process, studying how the stress-energy tensor evolves in time and approaches its late time behavior can provide insight into what is likely to happen in a more realistic model.

The method we have developed works in the region outside both the null shell and the event horizon. In the region outside the shell, Birkhoff's theorem ensures that the metric is that for Schwarzschild spacetime (2.2). In the region inside the shell the space is flat. Thus in both regions the mode equation for the quantum field is separable and inside the shell its solutions are known analytically. This allows for a numerical computation of the stress-energy 
tensor for the field in which only ordinary differential equations need to be solved numerically.

For the collapsing null shell model, the initial vacuum state of the quantum field is well defined and the main complication that occurs is due to the propagation of the modes across the null shell surface. The crux of our method involves the expansions of the in modes in terms of a complete set of solutions to the mode equation in the region outside the shell.

The stress-energy tensor for the quantum field is obtained by expanding the quantum field in terms of a complete set of modes. This expansion is substituted into the formula for the stress-energy tensor of the corresponding classical field and the expectation value is computed. If the field is in the in vacuum state then the result is an expression which involves sums and integrals over the mode functions for the in state and their derivatives. After the renormalization counterterms are subtracted off, the resulting stress-energy tensor is finite and can be computed. This is straightforward inside the null shell since the mode functions are known analytically and for the in state, the result is that the stress-energy tensor is equal to zero.

Outside the null shell and the event horizon the in modes do not assume a simple form in 4D. One approach to computing them would be to use the analytically known values for the modes inside the shell and on past null infinity to provide initial data for a numerical integration of the mode equation in the region exterior to the shell. However, outside the shell the in modes will not factorize into a product of a function that depends only on time and a function that depends only on the radial coordinate $r$. Thus the part of the mode equation that depends on both $r$ and $t$ must be solved numerically.

We have developed an alternative method which involves expanding each of the in modes in terms of a complete set of modes in Schwarzschild spacetime. The radial parts of these modes and the matching parameters must be computed numerically. The mode matching has been tested in the 2D case where there is no effective potential in the mode equation. It has also been partially tested for spherically symmetric modes in 4D both when the effective potential is modeled as a delta function and when the exact effective potential is used.

One advantage of the first method is that there are no matching parameters. A disadvantage is that one must solve a partial differential equation directly using numerical techniques. Conversely the chief advantage of the method developed here is that one only needs to numerically solve the radial mode equation, which is an ordinary differential equation. A second advantage is that the properties of the solutions to this equation are well understood. One disadvantage of our method is that the formulas for the matching parameters involve certain integrals that must be computed numerically. A second disadvantage is that the computation of the stress-energy tensor involves the numerical computation of triple integrals rather than single integrals over various products of the mode functions and their derivatives. It is not obvious to us which approach is more efficient. However, since no full numerical computation of the stress-energy tensor has been previously done for a quantized scalar field in a 4D spacetime where a black hole forms from collapse, we think the most important thing is to develop one viable method to do the calculation and that is what we present here.

When the expansions for the in modes are substituted into the formula for the unrenormalized stress-energy tensor one finds a combination of sums and integrals over various combinations of the modes and their derivatives. Renormalization of the stress-energy tensor can be accomplished by subtracting the corresponding expression that occurs in Schwarzschild spacetime for the Unruh state [26], adding that expression back and subtracting the renormalization counterterms. The result is the sum of two finite tensors. The first is the difference between the expressions for the unrenormalized stress-energy tensors in the in state and the Unruh state. The second is the renormalized stress-energy tensor for the Unruh state. The latter has been numerically computed for the masslesss minimally coupled scalar field in $[13,14]$. Thus one can simply add that result to the difference between the two stress-energy tensors to obtain the full renormalized stress-energy tensor for the scalar field in the in state in the collapsing null shell spacetime. This type of renormalization scheme has been used to compute the stress-energy tensors in Schwarzschild spacetime in the Unruh state for the conformally coupled massless scalar field [5,27] and for the massless spin 1 field [5]. It has also been used to compute a late time approximation to $\left\langle T_{t r}\right\rangle$ for the case of a massive minimally coupled scalar field in a spacetime consisting of a massive thin shell that is initially static and then collapses to form a black hole [28].

We have tested our method by numerically computing the difference between the stress-energy tensor for the in state in the collapsing null shell spacetime and the stressenergy tensor for the Unruh state in 2D. The results are compared with an analytic expression for the difference obtained from previous analytic calculations of the stressenergy tensor for the Unruh state [29] and the in vacuum state for the collapsing null shell spacetime [20,24]. Our results are in agreement with those calculations.

In Sec. II we introduce the collapsing null shell model and then discuss the modes for a massless minimally coupled scalar field in the null shell spacetime. A description of the method of computing the stress-energy tensor is given in Sec. III. Various mode functions in Schwarzschild spacetime that are used in the computation of the stressenergy tensor are discussed in Sec. IV. In Sec. V general expressions for the matching coefficients in the 4D case are derived followed by examples where the matching method is tested. Formulas needed for the computation of the 
stress-energy tensor in the 4D case are derived in the first part of Sec. VI. In the second part, the difference between the stress-energy tensor in the in vacuum state and the Unruh state is numerically computed for the $2 \mathrm{D}$ case and compared with the difference obtained from previous analytic calculations. Section VII contains a summary of our results. The appendixes contain some details of a proof and some derivations that are used in the $2 \mathrm{D}$ examples in Secs. V and VI. Throughout the paper, we use the sign conventions of [30] and units are chosen such that $\hbar=c=G=1$.

\section{MASSLESS MINIMALLY COUPLED SCALAR FIELD IN A SPACETIME WITH A COLLAPSING NULL SHELL}

\section{A. Collapsing null shell model}

We consider a model in which a spherically symmetric black hole forms from the collapse of a null shell. Our analysis of the spactime follows that in [20]. The metric inside the shell is the flat space metric

$$
d s^{2}=-d t^{2}+d r^{2}+r^{2} d \Omega^{2}
$$

and, by Birkhoff's theorem, the metric outside the shell is the Schwarzschild metric

$d s^{2}=-\left(1-\frac{2 M}{r}\right) d t_{s}^{2}+\left(1-\frac{2 M}{r}\right)^{-1} d r^{2}+r^{2} d \Omega^{2}$.

The two metrics need to be matched along the trajectory of the null shell. An obvious way to do this is to let the angular coordinates be continuous across the shell along with the radial coordinate $r$ that is related to the area of a two-sphere. Then the time coordinate is not continuous across the shell trajectory which is why we distinguish in the above metrics between the time coordinate $t$ inside the shell and the time coordinate $t_{s}$ outside the shell.

The actual matching is easier in terms of radial null coordinates which can be defined inside the shell as

$$
\begin{aligned}
& u=t-r, \\
& v=t+r,
\end{aligned}
$$

and outside the shell as

$$
\begin{gathered}
u_{s}=t_{s}-r_{*}, \\
v=t_{s}+r_{*},
\end{gathered}
$$

where

$$
r_{*}=r+2 M \log \left(\frac{r-2 M}{2 M}\right)
$$

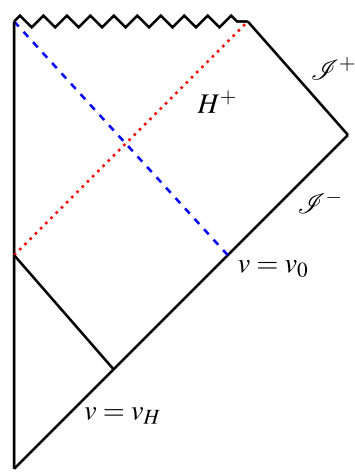

FIG. 1. Penrose diagram for a spacetime in which a null shell collapses to form a spherically symmetric black hole. The vertical line on the left corresponds to the surface $r=0$ which is also the surface where $u=v$. The trajectory of the shell (dashed blue curve) is $v=v_{0}$. The horizon, $H^{+}$, is the dotted red curve. Inside the shell trajectory it corresponds to the surface $u=v_{H}$ and outside the shell trajectory it corresponds to $u_{s}=\infty$.

is the usual tortoise coordinate in Schwarzschild spacetime. It is easiest to let $v$ be continuous across the shell trajectory which is denoted as $v=v_{0}$. The outgoing radial null coordinate is then discontinuous across the shell trajectory which is why it is denoted as $u$ inside the shell and $u_{s}$ outside. The relationship between $u$ and $u_{s}$ is $[20,21]$

$$
u_{s}=u-4 M \log \left(\frac{v_{H}-u}{4 M}\right)
$$

with

$$
v_{H} \equiv v_{0}-4 M .
$$

Note that the value of the flat space coordinate $u$ on the event horizon is $v_{H}$ as can be seen from Fig. 1. Inverting, one finds that [22]

$$
u=v_{H}-4 M W\left[\exp \left(\frac{v_{H}-u_{s}}{4 M}\right)\right],
$$

with $W$ the Lambert $\mathrm{W}$ function. A Penrose diagram for the resulting spacetime is sketched in Fig. 1.

\section{B. Massless minimally coupled scalar field}

The type of quantum field we consider is a massless minimally coupled scalar field which in a general spacetime satisfies the wave equation

$$
\square \phi=0 .
$$

In the null shell spacetime the field can be expanded in terms of a complete set of modes such that

$\phi=\sum_{\ell=0}^{\infty} \sum_{m=-\ell}^{\ell} \int_{0}^{\infty} d \omega\left[a_{\omega \ell m} f_{\omega \ell m}+a_{\omega \ell m}^{\dagger} f_{\omega \ell m}^{*}\right]$, 
with $a_{\omega \ell m}$ an annihilation operator. The modes are solutions to (2.9) which have the form

$$
f_{\omega \ell m}=N \frac{Y_{\ell, m}(\theta, \phi)}{r} \psi_{\omega \ell}(\tau, r)
$$

with $N$ a normalization constant and $\tau=t$ inside the shell trajectory and $\tau=t_{s}$ outside. Inside the shell trajectory the equation for $\psi_{\omega \ell}$ is

$$
-\frac{\partial^{2} \psi_{\omega \ell}}{\partial t^{2}}+\frac{\partial^{2} \psi_{\omega \ell}}{\partial r^{2}}-\frac{\ell(\ell+1)}{r^{2}} \psi_{\omega \ell}=0,
$$

while outside the shell the equation is

$$
-\frac{\partial^{2} \psi_{\omega \ell}}{\partial t_{s}^{2}}+\frac{\partial^{2} \chi_{\omega \ell}}{\partial r_{*}^{2}}-\left(1-\frac{2 M}{r}\right)\left(\frac{2 M}{r^{3}}+\frac{\ell(\ell+1)}{r^{2}}\right) \psi_{\omega \ell}=0 .
$$

The in vacuum state is defined by requiring that on $\mathcal{I}^{-}$

$$
\psi_{\omega \ell}^{\text {in }}=e^{-i \omega v} .
$$

The modes must also be regular on the surface $r=0$ inside the shell trajectory which implies that $\psi_{\omega \ell}^{\text {in }}=0$ there.

The normalization constant $N$ is fixed using the scalar product which is defined by the relation

$$
\left(f_{1}, f_{2}\right)=-i \int_{\Sigma} d \Sigma n^{\mu}\left[f_{1}(x) \stackrel{\leftrightarrow}{\partial_{\mu}} f_{2}^{*}(x)\right]
$$

Here $n^{\mu}$ is a future-directed unit vector orthogonal to the spacelike (or null) hypersurface $\Sigma$ and $d \Sigma$ is the volume element in $\Sigma$. The hypersurface $\Sigma$ is taken to be a Cauchy surface. To normalize the in modes it is easiest to use past null infinity, $\mathcal{I}^{-}$, as the Cauchy surface. If the orthonormal condition

$$
\left(f_{\omega \ell m}, f_{\omega^{\prime} \ell^{\prime} m^{\prime}}\right)=\delta_{\ell, \ell^{\prime}} \delta_{m, m^{\prime}} \delta\left(\omega-\omega^{\prime}\right)
$$

is imposed then it is straightforward to show that

$$
N=\frac{1}{\sqrt{4 \pi \omega}} .
$$

For the modes in the in state in the region inside the null shell trajectory

$$
\psi_{\omega \ell}^{\mathrm{in}}=e^{-i \omega t} \chi_{\omega \ell}^{\mathrm{in}}(r)
$$

Substituting this into (2.12) gives

$$
\frac{d^{2} \chi_{\omega \ell}^{\mathrm{in}}}{d r^{2}}=-\left[\omega^{2}-\frac{\ell(\ell+1)}{r^{2}}\right] \chi_{\omega \ell} .
$$

The solution for which $\psi_{\omega \ell}^{\mathrm{in}}$ vanishes at $r=0$ is

$$
\psi_{\omega \ell}^{\mathrm{in}}=C_{\ell} e^{-i \omega t} \omega r j_{\ell}(\omega r)
$$

where $C_{\ell}$ is a normalization constant and $j_{\ell}$ is a spherical Bessel function. The condition (2.14) on $\mathcal{I}^{-}$fixes the value of $C_{\ell}$. For example, for $\ell=0$, it is easy to show that $C_{0}=$ $-2 i$ and

$$
\psi_{\omega 0}^{\mathrm{in}}=e^{-i \omega v}-e^{-i \omega u} .
$$

In the region outside of the null shell trajectory $v=v_{0}$, the in modes still have the boundary condition (2.14). However, their other boundary condition is that $\psi_{\omega \ell}^{\mathrm{in}}$ and its first derivatives must be continuous across $v=v_{0}$. The fact that the time coordinates are different on either side of this surface makes it impossible to have a solution of the form $\psi_{\omega \ell}^{\text {in }}=e^{-i \omega t_{s}} \chi_{\omega \ell}^{\text {in }}(r)$ outside the null shell trajectory. However, it is possible to write $\psi_{\omega \ell}^{\text {in }}$ in terms of a complete set of mode functions of the form $\psi_{\omega \ell}=e^{-i \omega t_{s}} \chi_{\omega \ell}(r)$ outside the null shell trajectory as is shown in Sec. V.

\section{METHOD TO COMPUTE THE STRESS-ENERGY TENSOR}

The stress-energy tensor for the quantized massless minimally coupled scalar field, $\left\langle T_{a b}\right\rangle$, is to be computed for the in vacuum state in the region outside the null shell and outside the event horizon. The stress-energy tensor for the classical field is

$$
T_{a b}=\partial_{a} \Phi \partial_{b} \Phi-\frac{1}{2} g_{a b} g^{c d} \partial_{c} \Phi \partial_{d} \Phi .
$$

To compute $\left\langle\right.$ in $\left|T_{a b}\right|$ in $\rangle$, one can substitute (2.10) into (3.1), use the complete set of modes for the in vacuum state $f_{\omega \ell m}^{\text {in }}$, and compute the expectation value. There are two things which make this difficult. One is computing the modes $f_{\omega \ell m}^{\text {in }}$ in the region outside the shell and the other is renormalizing the stress-energy tensor. Our method to compute the stress-energy tensor provides one way to overcome these difficulties.

First, we renormalize by subtracting from the unrenormalized expression for the stress-energy tensor for the in vacuum state, the unrenormalized stress-energy tensor for the Unruh state. Since the renormalization counterterms are local and thus do not depend on the state of the quantum field, this quantity will be finite. Then we add back the unrenormalized stress-energy tensor for the Unruh state and then subtract from it the renormalization counter terms. Schematically one can write

$$
\begin{aligned}
\left\langle\text { in }\left|T_{a b}\right| \text { in }\right\rangle_{\text {ren }} & =\Delta\left\langle T_{a b}\right\rangle+\left\langle U\left|T_{a b}\right| U\right\rangle_{\text {ren }}, \\
\Delta\left\langle T_{a b}\right\rangle & =\left\langle\text { in }\left|T_{a b}\right| \text { in }\right\rangle_{\text {unren }}-\left\langle U\left|T_{a b}\right| U\right\rangle_{\text {unren }} .
\end{aligned}
$$


The quantity $\left\langle U\left|T_{a b}\right| U\right\rangle_{\text {ren }}$ has been numerically computed for a massless minimally coupled scalar field in Schwarzschild spacetime $[13,14]$. Thus what remains is to compute the difference between the unrenormalized expressions. To do that it is necessary to discuss the computation of the mode functions for the quantum field that are relevant for the in and Unruh states. It is worth pointing out that the computation of $\left\langle U\left|T_{a b}\right| U\right\rangle_{\text {ren }}$ done in $[13,14]$ was done for pure Schwarzschild spacetime outside the event horizon. However the computation we wish to do for $\left\langle\operatorname{in}\left|T_{a b}\right| \text { in }\right\rangle_{\text {ren }}$ is for the null shell spacetime outside both the shell and the horizon. The reason that there is no problem is that the renormalization counterterms are local and so are the same in this part of the null shell spacetime as they are in pure Schwarzschild spacetime.

Analytic expressions for the mode functions in the in vacuum state, $f_{\omega \ell m}^{\text {in }}$, inside the shell are given in (2.20). However, it is not easy to continue these to the region outside the shell because the time coordinate $t$ and the right moving radial null coordinate $u$ are not continuous across the shell. However, the known solutions inside the null shell along with their behavior on $\mathcal{I}^{-}$can be used to fix the initial data on a Cauchy surface in the null shell spacetime. The Cauchy surface we consider here, consists of the part of $\mathcal{I}^{-}$with $v_{0} \leq v<\infty$ along with the trajectory of the null shell. This initial data could be used for a numerical calculation of the partial differential equation satisfied by $f_{\omega \ell m}^{\text {in }}$ outside the shell. Alternatively, one can expand $f_{\omega \ell m}^{\text {in }}$ in terms of a complete set of modes in the region outside the shell and use the data on the Cauchy surface to determine the matching coefficients.

Here we take a variation of the latter approach by noting that the spacetime geometry outside the shell is the Schwarzschild geometry. Because of this, it is possible to do the matching in the corresponding part of Schwarzschild spacetime. The advantage of this is that the matching can be to a complete set of modes in the region outside the horizon in Schwarzschild spacetime. These modes are well understood and straightforward to work with numerically. The disadvantage is that the relevant part of the Cauchy surface in the null shell spacetime discussed above does not form a Cauchy surface in the Schwarzschild spacetime. This can be remedied by adding a segment along the future horizon with $-\infty<v \leq v_{0}$. The result is a Cauchy surface for the part of Schwarzschild spacetime that is outside of the past and future horizons. It is illustrated in Fig. 2. It is worth noting that the part of the Cauchy surface on the future horizon is not causally connected with the region outside the future horizon and outside the surface $v=v_{0}$. The corresponding region in the null shell spacetime is the region where we want to compute the stress-energy tensor. Thus any initial data can be used for the mode function $f_{\omega \ell m}^{\text {in }}$ on that surface so long as $f_{\omega \ell m}^{\mathrm{in}}$ is continuous at the point where the future horizon intersects the part of the Cauchy surface with $v=v_{0}$.

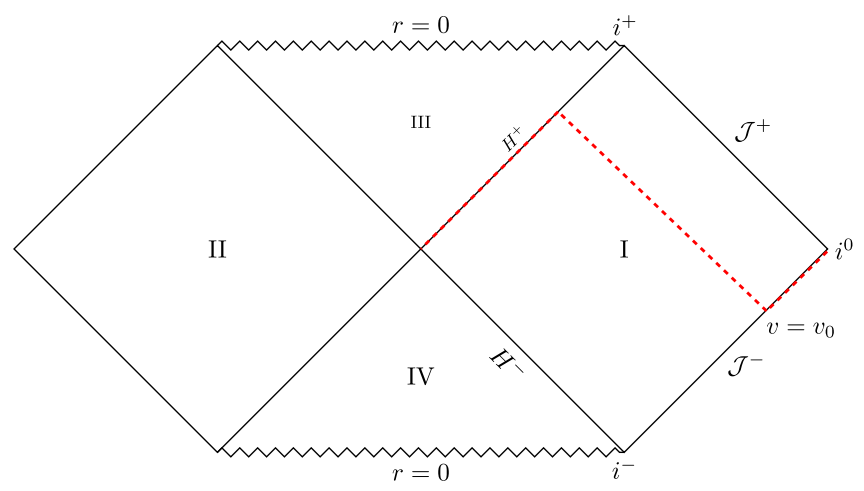

FIG. 2. Penrose diagram for Schwarzschild spacetime showing the Cauchy surface used for matching the in modes in the null shell spacetime to a complete set of modes in Schwarzschild spacetime in the region outside the past and future horizons. The Cauchy surface is denoted by the dashed red curve.

\section{COMPLETE SETS OF MODE FUNCTIONS IN SCHWARZSCHILD SPACETIME}

In this paper we work with four complete sets of mode functions for the part of Schwarzschild spacetime that is outside both the past and future horizons. The frequencies of all of the modes that we consider are taken to be non-negative.

\section{A. Modes used for the in state}

To expand the modes for the in state in terms of a complete set of modes in Schwarzschild spacetime we find it most convenient to choose the complete sets of modes that consists of the union of modes that are positive frequency on the future horizon $\mathrm{H}^{+}$and zero on future null infinity, $\mathcal{I}^{+}$(labeled by $f_{\omega \ell m}^{H^{+}}$), and modes that are positive frequency on $\mathcal{I}^{+}$and zero on $H^{+}$(labeled by $f_{\omega \ell m}^{\mathcal{I}^{+}}$). Both sets of modes are of the general form

$$
\psi_{\omega \ell}=e^{-i \omega t_{s}} \chi_{\omega \ell}(r),
$$

with $0 \leq \omega<\infty$. Substituting into (2.13) gives the radial mode equation for Schwarzschild spacetime

$\frac{d^{2} \chi_{\omega \ell}}{d r_{*}^{2}}=-\left[\omega^{2}-\left(1-\frac{2 M}{r}\right)\left(\frac{2 M}{r^{3}}+\frac{\ell(\ell+1)}{r^{2}}\right)\right] \chi_{\omega \ell}$.

These modes are normalized on the Cauchy surface consisting of $\mathrm{H}^{+}$and $\mathcal{I}^{+}$with the result (2.17).

It is useful to consider a different complete set of mode functions of the form (4.1) which are defined by two linearly independent solutions to the radial mode equation (4.2) with the properties

$$
\chi_{R}^{\infty} \rightarrow e^{i \omega r_{*}}, \quad r_{*} \rightarrow \infty,
$$




$$
\chi_{L}^{\infty} \rightarrow e^{-i \omega r_{*}}, \quad r_{*} \rightarrow \infty .
$$

Near the event horizon they have the behaviors [31]

$\chi_{R}^{\infty} \rightarrow E_{R}(\omega) e^{i \omega r_{*}}+F_{R}(\omega) e^{-i \omega r_{*}}, \quad r_{*} \rightarrow-\infty$,

$\chi_{L}^{\infty} \rightarrow E_{L}(\omega) e^{i \omega r_{*}}+F_{L}(\omega) e^{-i \omega r_{*}}, \quad r_{*} \rightarrow-\infty$,

where $E_{R}, E_{L}, F_{R}$, and $F_{L}$ are scattering parameters that can be determined numerically. They satisfy the relation $E_{R} F_{L}-E_{L} F_{R}=1{ }^{1}$

For the modes $f_{\omega \ell m}^{H^{+}}$, on the future horizon $\psi_{\omega \ell}^{H^{+}}=e^{-i \omega v}$ while $\psi_{\omega \ell}^{H^{+}}=0$ on $\mathcal{I}^{+}$. The radial mode function which has these properties is

$$
\chi_{\omega \ell}^{H^{+}}=\frac{1}{F_{L}} \chi_{L}^{\infty} .
$$

This is easily verified by evaluating the resulting mode function $\psi_{\omega \ell}^{H^{+}}$on $H^{+}$and $\mathcal{I}^{+}$. To see how this works consider the behavior near $\mathcal{I}^{+}$:

$$
\psi_{\omega \ell}^{H^{+}} \rightarrow \frac{1}{F_{L}} e^{-i \omega v-\epsilon v} \rightarrow 0, \quad v \rightarrow \infty .
$$

Here we have used a positive integrating factor $\epsilon$ to explicitly show that this mode function vanishes on $\mathcal{I}^{+}$ where $v=\infty$.

For the modes $f_{\omega \ell m}^{\mathcal{I}^{+}}$, on $H^{+}, \psi_{\omega \ell}^{\mathcal{I}^{+}}=0$ while on $\mathcal{I}^{+}$, $\psi_{\omega \ell}^{\mathcal{I}^{+}} \rightarrow e^{-i \omega u_{s}}$. The radial mode function which results in these properties is

$$
\chi_{\omega \ell}^{\mathcal{I}^{+}}=\chi_{R}^{\infty}-\frac{F_{R}}{F_{L}} \chi_{L}^{\infty} .
$$

\section{B. Complete sets of modes used to define the Unruh state}

Before discussing the modes that can be used to define the Unruh state, it is useful to consider a complete set of mode functions that are positive frequency on either the past horizon $H^{-}$and vanish on $\mathcal{I}^{-}$(denoted by $f_{\omega \ell m}^{H^{-}}$) or which vanish on $H^{-}$and are positive frequency on $\mathcal{I}^{-}$ (denoted by $f_{\omega \ell m}^{\mathcal{I}^{-}}$). For the modes $f_{\omega \ell m}^{H^{-}}$, on $H^{-}, \psi_{\omega \ell}^{H^{-}}=$ $e^{-i \omega u_{s}}$ while $\psi_{\omega \ell}^{H^{-}}=0$ on $\mathcal{I}^{-}$. The radial mode function which has these properties is [31]

$$
\chi_{\omega \ell}^{H^{-}}=\frac{\chi_{R}^{\infty}}{E_{R}} .
$$

For the modes $f_{\omega \ell m}^{\mathcal{I}^{-}}$, on $H^{-}, \psi_{\omega \ell}^{\mathcal{I}^{-}}=0$ while on $\mathcal{I}^{-}$, $\psi_{\omega \ell}^{\mathcal{I}^{-}} \rightarrow e^{-i \omega v}$. The radial mode functions which have these properties is [31]

\footnotetext{
${ }^{1}$ The subscripts $r$ and $l$ in [31] have been changed here to $R$ and $L$ respectively.
}

$$
\chi_{\omega \ell}^{\mathcal{I}^{-}}=\chi_{L}^{\infty}-\frac{E_{L}}{E_{R}} \chi_{R}^{\infty} .
$$

These modes are normalized on the Cauchy surface consisting of $\mathrm{H}^{-}$and $\mathcal{I}^{-}$with the result (2.17).

The Unruh state in Schwarzschild spacetime consists of a complete set of modes consisting of the modes $f_{\omega \ell m}^{\mathcal{I}^{-}}$and the modes (denoted by $f_{\omega \ell m}^{K}$ ) that on $H^{-}$have the form

$$
\psi_{\omega_{K} \ell}^{K}=e^{-i \omega_{K} U},
$$

with

$$
U=-\frac{e^{-\kappa u}}{\kappa} .
$$

Here $\kappa=(4 M)^{-1}$ is the surface gravity of the black hole and $0 \leq \omega_{K}<\infty$. These modes vanish on $\mathcal{I}^{-}$and can be normalized on the Cauchy surface that is the union between $H^{-}$and $\mathcal{I}^{-}$with the result that ${ }^{2}$

$$
f_{\omega \ell m}^{K}=\frac{Y_{\ell m}}{\sqrt{4 \pi \omega_{K}} r} \psi_{\omega \ell}^{K} .
$$

They can be expanded in terms of the $f_{\omega \ell m}^{H^{-}}$modes. The result is given in (5.16) and (5.17).

Since the method used to compute the stress-energy tensor involves subtracting the unrenormalized stressenergy tensor for the Unruh state it is useful to write the modes associated with this state, $f_{\omega \ell m}^{H^{-}}$and $f_{\omega \ell m}^{\mathcal{I}^{-}}$, in terms of $f_{\omega \ell m}^{H^{+}}$and $f_{\omega \ell m}^{\mathcal{I}^{+}}$. The result is

$$
\begin{aligned}
& f_{\omega \ell m}^{H^{-}}=\frac{1}{E_{R}}\left(F_{R} f_{\omega \ell m}^{H^{+}}+f_{\omega \ell m}^{\mathcal{I}^{+}}\right), \\
& f_{\omega \ell m}^{\mathcal{I}^{-}}=\frac{1}{E_{R}}\left(f_{\omega \ell m}^{H^{+}}-E_{L} f_{\omega \ell m}^{\mathcal{I}^{+}}\right) .
\end{aligned}
$$

\section{MATCHING COEFFICIENTS}

\section{A. General formulas}

In this section general formulas are derived for the matching coefficients used in an expansion of the modes of a massless minimally coupled scalar field for the in vacuum state in the collapsing null shell spacetime in terms of a complete set of modes in Schwarzschild spacetime in the region outside the past and future horizons. These can be used in the computation of the stress-energy tensor, $\left\langle\right.$ in $\left|T_{a b}\right|$ in $\rangle$, for the scalar field in the part of the collapsing null shell spacetime that is outside of the shell and outside of the event horizon.

\footnotetext{
${ }^{2}$ Here we use the entire surface $H^{-}$which extends from $U=$ $-\infty$ to $U=+\infty$.
} 
The expansion of the in mode functions has the form

$$
\begin{aligned}
f_{\omega \ell m}^{\text {in }}= & \sum_{\ell^{\prime}=0}^{\infty} \sum_{m^{\prime}=-\ell^{\prime}}^{\ell^{\prime}} \int_{0}^{\infty} d \omega^{\prime}\left[A_{\omega \ell m \omega^{\prime} \ell^{\prime} m^{\prime}}^{\mathcal{I}^{+}} f_{\omega^{\prime} \ell^{\prime} m^{\prime}}^{\mathcal{I}^{+}}\right. \\
& +B_{\omega l m \omega^{\prime} \ell^{\prime} m^{\prime}}^{\mathcal{I}^{+}}\left(f_{\omega^{\prime} \ell^{\prime} m^{\prime}}^{\mathcal{I}^{+}}\right)^{*}+A_{\omega \ell m \omega^{\prime} \ell^{\prime} m^{\prime}}^{H^{+}} f_{\omega^{\prime} \ell^{\prime} m^{\prime}}^{H^{+}} \\
& \left.+B_{\omega \ell m \omega^{\prime} \ell^{\prime} m^{\prime}}^{H^{+}}\left(f_{\omega^{\prime} \ell^{\prime} m^{\prime}}^{H^{+}}\right)^{*}\right] .
\end{aligned}
$$

The matching coefficients are found using the scalar product in (2.15) and the orthonormality of the modes $f^{\left(\mathcal{I}^{+}, H^{+}\right)}$with respect to this scalar product. The result is

$$
\begin{aligned}
& A_{\omega \ell m \omega^{\prime} \ell^{\prime} m^{\prime}}^{\left(\mathcal{I}^{+}, H^{+}\right)}=\left(f_{\omega \ell m}^{\text {in }}, f_{\omega^{\prime} \ell^{\prime} m^{\prime}}^{\left(\mathcal{I}^{+}, H^{+}\right)}\right), \\
& B_{\omega \ell m \omega^{\prime} \ell^{\prime} m^{\prime}}^{\left(\mathcal{I}^{+}, H^{+}\right)}=-\left(f_{\omega \ell m}^{\text {in }},\left(f_{\omega^{\prime} \ell^{\prime} m^{\prime}}^{\left(\mathcal{I}^{+}, H^{+}\right)}\right)^{*}\right) .
\end{aligned}
$$

For the Cauchy surface we consider, (2.15) reduces to integrals of the form

$$
\int d u \int d \Omega r^{2} \stackrel{\leftrightarrow}{\partial_{u}}, \quad \int d v \int d \Omega r^{2} \stackrel{\leftrightarrow}{\partial_{v}}
$$

On the hypersurfaces where these integrals are computed, the following properties for spherical harmonics can be used:

$$
\int d \Omega Y_{l m}(\theta, \phi) Y_{l^{\prime} m^{\prime}}^{*}(\theta, \phi)=\delta_{l, l^{\prime}} \delta_{m, m^{\prime}}
$$

$$
\int d \Omega Y_{l m}(\theta, \phi) Y_{l^{\prime} m^{\prime}}(\theta, \phi)=(-1)^{m} \delta_{l, l^{\prime}} \delta_{m,-m^{\prime}}
$$

As a result we can write

$$
\begin{aligned}
& A_{\omega l m \omega^{\prime} l^{\prime} m^{\prime}}^{\left(\mathcal{I}^{+}, H^{+}\right.}=\delta_{l, l^{\prime}} \delta_{m, m^{\prime}} A_{\omega \omega^{\prime} \ell}^{\left(\mathcal{I}^{+}, H^{+}\right)}, \\
& B_{\omega l m \omega^{\prime} l^{\prime} m^{\prime}}^{\left(\mathcal{I}^{+}, H^{+}\right)}=(-1)^{m} \delta_{l, l^{\prime}} \delta_{m,-m^{\prime}} B_{\omega \omega^{\prime} \ell}^{\left(\mathcal{I}^{+}, H^{+}\right)},
\end{aligned}
$$

and

$$
\begin{aligned}
f_{\omega \ell m}^{\text {in }}= & \frac{Y_{\ell m}}{r \sqrt{4 \pi}} \int_{0}^{\infty} \frac{d \omega^{\prime}}{\sqrt{\omega^{\prime}}}\left[A_{\omega \omega^{\prime} \ell}^{\mathcal{I}^{+}} \psi_{\omega^{\prime} \ell}^{\mathcal{I}^{+}}+B_{\omega \omega^{\prime} \ell}^{\mathcal{I}^{+}}\left(\psi_{\omega^{\prime} \ell}^{\mathcal{I}^{+}}\right)^{*}\right. \\
& \left.+A_{\omega \omega^{\prime} \ell}^{H^{+}} \psi_{\omega^{\prime} \ell}^{H^{+}}+B_{\omega \omega^{\prime} \ell}^{H^{+}}\left(\psi_{\omega^{\prime} \ell}^{H^{+}}\right)^{*}\right] .
\end{aligned}
$$

From this expression one can see that if, at small $\omega^{\prime}$, the matching coefficients go like $\frac{1}{\sqrt{\omega^{\prime}}}$ then there is an infrared divergence in the integral and it is not obvious how to deal with it. For this reason, we use integrations by parts in some of the computations of the matching coefficients below to avoid this difficulty. For Schwarzschild spacetime in 4D, our results when substituted into (5.6) do not give infrared divergences.

The contribution to the matching coefficients from the three segments of the Cauchy surface in Fig. 2 are

$$
\begin{aligned}
A_{\omega \omega^{\prime} l}^{\left(\mathcal{I}^{+}, H^{+}\right)} & =\left(A_{\omega \omega^{\prime} l}^{\left(\mathcal{I}^{+}, H^{+}\right)}\right)_{H^{+}}+\left(A_{\omega \omega^{\prime} l}^{\left(\mathcal{I}^{+} H^{+}\right)}\right)_{v_{0}}+\left(A_{\omega \omega^{\prime} l}^{\left(\mathcal{I}^{+}, H^{+}\right)}\right)_{\mathcal{I}^{-}}, \\
\left(A_{\omega \omega^{\prime} l}^{\left(\mathcal{I}^{+}, H^{+}\right)}\right)_{H^{+}} & =-\frac{i}{4 \pi \sqrt{\omega \omega^{\prime}}} \int_{-\infty}^{v_{0}} d v \psi_{\omega l}^{\text {in }}\left(u=v_{H}, v\right) \stackrel{\leftrightarrow}{\partial_{v}}\left[\psi_{\omega^{\prime} l}^{\left(\mathcal{I}^{+}, H^{+}\right)}\left(u_{s}=\infty, v\right)\right]^{*}, \\
\left(A_{\omega \omega^{\prime} l}^{\left(\mathcal{I}^{+}, H^{+}\right)}\right)_{v_{0}} & =-\frac{i}{4 \pi \sqrt{\omega \omega^{\prime}}} \int_{-\infty}^{v_{H}} d u \psi_{\omega l}^{\mathrm{in}}\left(u, v_{0}\right) \stackrel{\leftrightarrow}{\partial_{u}}\left[\psi_{\omega^{\prime} l}^{\left(\mathcal{I}^{+}, H^{+}\right)}\left(u_{s}(u), v_{0}\right)\right]^{*}, \\
\left(A_{\omega \omega^{\prime} l}^{\left(\mathcal{I}^{+}, H^{+}\right)}\right)_{\mathcal{I}^{-}} & =-\frac{i}{4 \pi \sqrt{\omega \omega^{\prime}}} \int_{v_{0}}^{\infty} d v \psi_{\omega l}^{\mathrm{in}}(u=-\infty, v) \stackrel{\leftrightarrow}{\partial_{v}}\left[\psi_{\omega^{\prime} l}^{\left(\mathcal{I}^{+}, H^{+}\right)}\left(u_{s}=-\infty, v\right)\right]^{*},
\end{aligned}
$$

and

$$
\begin{aligned}
B_{\omega \omega^{\prime} l}^{\left(\mathcal{I}^{+}, H^{+}\right)} & =\left(B_{\omega \omega^{\prime} l}^{\left(\mathcal{I}^{+}, H^{+}\right)}\right)_{H^{+}}+\left(B_{\omega \omega^{\prime} l}^{\left(\mathcal{I}^{+}, H^{+}\right)}\right)_{v_{0}}+\left(B_{\omega \omega^{\prime} l}^{\left(\mathcal{I}^{+}, H^{+}\right)}\right)_{\mathcal{I}^{-}}, \\
\left(B_{\omega \omega^{\prime} l}^{\left(\mathcal{I}^{+}, H^{+}\right)}\right)_{H^{+}} & =\frac{i}{4 \pi \sqrt{\omega \omega^{\prime}}} \int_{-\infty}^{v_{0}} d v \psi_{\omega l}^{\mathrm{in}}\left(u=v_{H}, v\right) \stackrel{\leftrightarrow}{\partial_{v}} \psi_{\omega^{\prime} l}^{\left(\mathcal{I}^{+}, H^{+}\right)}\left(u_{s}=\infty, v\right), \\
\left(B_{\omega \omega^{\prime} l}^{\left(\mathcal{I}^{+}, H^{+}\right)}\right)_{v_{0}} & =\frac{i}{4 \pi \sqrt{\omega \omega^{\prime}}} \int_{-\infty}^{v_{H}} d u \psi_{\omega l}^{\mathrm{in}}\left(u, v_{0}\right) \stackrel{\leftrightarrow}{\partial_{u}} \psi_{\omega^{\prime} l}^{\left(\mathcal{I}^{+}, H^{+}\right)}\left(u_{s}(u), v_{0}\right), \\
\left(B_{\omega \omega^{\prime} l}^{\left(\mathcal{I}^{+}, H^{+}\right)}\right)_{\mathcal{I}^{-}} & =\frac{i}{4 \pi \sqrt{\omega \omega^{\prime}}} \int_{v_{0}}^{\infty} d v \psi_{\omega l}^{\text {in }}(u=-\infty, v) \stackrel{\leftrightarrow}{\partial_{v}} \psi_{\omega^{\prime} l}^{\left(\mathcal{I}^{+}, H^{+}\right)}\left(u_{s}=-\infty, v\right) .
\end{aligned}
$$


It is important to note that the integrals in (5.7b) and (5.8b) are computed with the integrands evaluated on $\mathrm{H}^{+}$. Since $\psi_{\omega^{\prime} l}^{\mathcal{I}^{+}}=0$ on $\mathrm{H}^{+}$

$$
\left(A_{\omega \omega^{\prime} l}^{\mathcal{I}^{+}}\right)_{H^{+}}=\left(B_{\omega \omega^{\prime} l}^{\mathcal{I}^{+}}\right)_{H^{+}}=0 .
$$

On $H^{+}, \psi_{\omega \ell}^{H^{+}}=e^{-i \omega v}$. The part of the Cauchy surface in Schwarzschild spacetime which is on $\mathrm{H}^{+}$has no counterpart in the collapsing null shell spacetime and, as discussed in Sec. III, is causally disconnected from the region outside the collapsing null shell and outside the event horizon. Thus the only restriction on the mode functions $\psi_{\omega \ell}^{\text {in }}$ for this surface is continuity at $v=v_{0}$. The simplest mode function to use on this surface is then

$$
\psi_{\omega \ell}^{\text {in }}\left(u=v_{H}, v\right)=\psi_{\omega \ell}^{\text {in }}\left(u=v_{H}, v_{0}\right) .
$$

With this choice it turns out to be useful to write the contribution to the matching coefficients from $\mathrm{H}^{+}$in the form

$$
\begin{aligned}
& \left(A_{\omega \omega^{\prime} l}^{H^{+}}\right)_{H^{+}}=\frac{i}{4 \pi \sqrt{\omega \omega^{\prime}}} \psi_{\omega \ell}^{\text {in }}\left(v_{H}, v_{0}\right) e^{i \omega^{\prime} v_{0}}-\frac{i}{2 \pi} \sqrt{\frac{\omega^{\prime}}{\omega}} \frac{e^{i \omega^{\prime} v_{0}}}{\omega^{\prime}-i \epsilon} \psi_{\omega \ell}^{\text {in }}\left(v_{H}, v_{0}\right), \\
& \left(B_{\omega \omega^{\prime} l}^{H^{+}}\right)_{H^{+}}=-\frac{i}{4 \pi \sqrt{\omega \omega^{\prime}}} \psi_{\omega \ell}^{\text {in }}\left(v_{H}, v_{0}\right) e^{-i \omega^{\prime} v_{0}}+\frac{i}{2 \pi} \sqrt{\frac{\omega^{\prime}}{\omega}} \frac{e^{-i \omega^{\prime} v_{0}}}{\omega^{\prime}+i \epsilon} \psi_{\omega \ell}^{\text {in }}\left(v_{H}, v_{0}\right),
\end{aligned}
$$

where for each integral an integration by parts has been done and an integrating factor $0<\epsilon \ll 1$ has been included to make the integrals converge.

The integrals in (5.7c) and (5.8c) are computed with the integrands evaluated on the surface $v=v_{0}$. In this case $\psi_{\omega \ell}^{\mathrm{in}}$ is given by (2.20) while analytic expressions for $\psi_{\omega \ell}^{\mathcal{I}^{+}, H^{+}}$are only known for the limits $u_{s} \rightarrow \pm \infty$. For intermediate values of $u_{s}$ these modes must be computed numerically.

The integrals in (5.7d) and (5.8d) are computed with the integrands evaluated on the surface $\mathcal{I}^{-}$. In this case $\psi_{\omega \ell}^{\text {in }}$ is given by (2.14). From (4.5) and (4.7) one can deduce that on $\mathcal{I}^{-}$

$$
\begin{aligned}
\psi_{\omega \ell}^{H^{+}} & =\frac{1}{F_{L}} e^{-i \omega v}, \\
\psi_{\omega \ell}^{\mathcal{I}^{+}} & =-\frac{F_{R}}{F_{L}} e^{-i \omega v} .
\end{aligned}
$$

To avoid infrared divergences in (5.6) it is useful to subtract and then add back the quantity $e^{-i \omega v_{0}}$ from $\psi_{\omega \ell}^{\text {in }}$ in $(5.7 \mathrm{c})$ and $(5.8 \mathrm{c})$. Then after integrations by parts the contributions from the surface $v=v_{0}$ can be written as

$$
\begin{aligned}
\left(A_{\omega \omega^{\prime} l}^{H^{+}}\right)_{v_{0}}= & -\frac{i}{4 \pi \sqrt{\omega \omega^{\prime}}} \psi_{\omega \ell}^{\text {in }}\left(v_{H}, v_{0}\right) e^{i \omega^{\prime} v_{0}}+\frac{i}{4 \pi \sqrt{\omega \omega^{\prime}} F_{L}^{*}\left(\omega^{\prime}, \ell\right)} e^{-i\left(\omega-\omega^{\prime}\right) v_{0}} \\
& +\frac{i}{2 \pi \sqrt{\omega \omega^{\prime}}} \int_{-\infty}^{v_{H}} d u\left[\partial_{u} \psi_{\omega \ell}^{\text {in }}\left(u, v_{0}\right)\right] \psi_{\omega^{\prime} \ell}^{H^{+} *}\left(u_{s}(u), v_{0}\right), \\
\left(B_{\omega \omega^{\prime} l}^{H^{+} l}\right)_{v_{0}}= & \frac{i}{4 \pi \sqrt{\omega \omega^{\prime}}} \psi_{\omega \ell}^{\text {in }}\left(v_{H}, v_{0}\right) e^{-i \omega^{\prime} v_{0}}-\frac{i}{4 \pi \sqrt{\omega \omega^{\prime}} F_{L}\left(\omega^{\prime}, \ell\right)} e^{-i\left(\omega+\omega^{\prime}\right) v_{0}} \\
& -\frac{i}{2 \pi \sqrt{\omega \omega^{\prime}}} \int_{-\infty}^{v_{H}} d u\left[\partial_{u} \psi_{\omega \ell}^{\text {in }}\left(u, v_{0}\right)\right] \psi_{\omega^{\prime} \ell}^{H^{+}}\left(u_{s}(u), v_{0}\right), \\
\left(A_{\omega \omega^{\prime} l}^{\mathcal{I}^{+}}\right)_{v_{0}}=-\frac{i}{4 \pi \sqrt{\omega \omega^{\prime}}} & \frac{F_{R}^{*}\left(\omega^{\prime}, \ell\right)}{F_{L}^{*}\left(\omega^{\prime}, \ell\right)} e^{-i\left(\omega-\omega^{\prime}\right) v_{0}}-\frac{i}{2 \pi \sqrt{\omega \omega^{\prime}}} \int_{-\infty}^{v_{H}} d u\left[\psi_{\omega \ell}^{\text {in }}\left(u, v_{0}\right)-e^{-i \omega v_{0}}\right] \partial_{u} \psi_{\omega^{\prime} \ell}^{\mathcal{I}^{+} *}\left(u_{s}(u), v_{0}\right), \\
\left(B_{\omega \omega^{\prime} l}^{\mathcal{I}^{+}}\right)_{v_{0}}=\frac{i}{4 \pi \sqrt{\omega \omega^{\prime}}} & \frac{F_{R}\left(\omega^{\prime}, \ell\right)}{F_{L}\left(\omega^{\prime}, \ell\right)} e^{-i\left(\omega+\omega^{\prime}\right) v_{0}}+\frac{i}{2 \pi \sqrt{\omega \omega^{\prime}}} \int_{-\infty}^{v_{H}} d u\left[\psi_{\omega \ell}^{\text {in }}\left(u, v_{0}\right)-e^{-i \omega v_{0}}\right] \partial_{u} \psi_{\omega^{\prime} \ell}^{\mathcal{I}^{+}}\left(u_{s}(u), v_{0}\right) .
\end{aligned}
$$

The modes on $\mathcal{I}^{-}$take on the simple forms (2.14) and (5.12). This makes it possible to evaluate the integrals for the contributions to the matching parameters from that surface. After integrating by parts, we find

$$
\left(A_{\omega \omega^{\prime} l}^{H^{+}}\right)_{\mathcal{I}^{-}}=-\frac{i}{4 \pi \sqrt{\omega \omega^{\prime}} F_{L}^{*}\left(\omega^{\prime}, \ell\right)} e^{-i\left(\omega-\omega^{\prime}\right) v_{0}}+\frac{i}{2 \pi} \sqrt{\frac{\omega^{\prime}}{\omega}} \frac{1}{F_{L}^{*}\left(\omega^{\prime}, \ell\right)} \frac{e^{i\left(\omega^{\prime}-\omega\right) v_{0}}}{\omega^{\prime}-\omega+i \epsilon},
$$




$$
\begin{aligned}
& \left(B_{\omega \omega^{\prime} l}^{H^{+}}\right)_{\mathcal{I}^{-}}=\frac{i}{4 \pi \sqrt{\omega \omega^{\prime}} F_{L}\left(\omega^{\prime}, \ell\right)} e^{-i\left(\omega+\omega^{\prime}\right) v_{0}}-\frac{i}{2 \pi} \sqrt{\frac{\omega^{\prime}}{\omega}} \frac{1}{F_{L}\left(\omega^{\prime}, \ell\right)} \frac{e^{-i\left(\omega+\omega^{\prime}\right) v_{0}}}{\omega^{\prime}+\omega-i \epsilon}, \\
& \left(A_{\omega \omega^{\prime} l}^{\mathcal{I}^{+}}\right)_{\mathcal{I}^{-}}=\frac{i}{4 \pi \sqrt{\omega \omega^{\prime}}} \frac{F_{R}^{*}\left(\omega^{\prime}, \ell\right)}{F_{L}^{*}\left(\omega^{\prime}, \ell\right)} e^{-i\left(\omega-\omega^{\prime}\right) v_{0}}-\frac{i}{2 \pi} \sqrt{\frac{\omega^{\prime}}{\omega}} \frac{F_{R}^{*}\left(\omega^{\prime}, \ell\right)}{F_{L}^{*}\left(\omega^{\prime}, \ell\right)} \frac{e^{-i\left(\omega-\omega^{\prime}\right) v_{0}}}{\omega^{\prime}-\omega+i \epsilon}, \\
& \left(B_{\omega \omega^{\prime} l}^{\mathcal{I}^{+}}\right)_{\mathcal{I}^{-}}=-\frac{i}{4 \pi \sqrt{\omega \omega^{\prime}}} \frac{F_{R}\left(\omega^{\prime}, \ell\right)}{F_{L}\left(\omega^{\prime}, \ell\right)} e^{-i\left(\omega+\omega^{\prime}\right) v_{0}}+\frac{i}{2 \pi} \sqrt{\frac{\omega^{\prime}}{\omega}} \frac{F_{R}\left(\omega^{\prime}, \ell\right)}{F_{L}\left(\omega^{\prime}, \ell\right)} \frac{e^{-i\left(\omega+\omega^{\prime}\right) v_{0}}}{\omega^{\prime}+\omega-i \epsilon} .
\end{aligned}
$$

Combining these results together, the general formulas for the matching coefficients are

$$
\begin{aligned}
& A_{\omega \omega^{\prime} l}^{H^{+}}=-\frac{i}{2 \pi} \sqrt{\frac{\omega^{\prime}}{\omega}} \frac{e^{i \omega^{\prime} v_{0}}}{\omega^{\prime}-i \epsilon} \psi_{\omega \ell}^{\text {in }}\left(v_{H}, v_{0}\right)+\frac{i}{2 \pi} \sqrt{\frac{\omega^{\prime}}{\omega}} \frac{1}{F_{L}^{*}\left(\omega^{\prime}, \ell\right)} \frac{e^{i\left(\omega^{\prime}-\omega\right) v_{0}}}{\omega^{\prime}-\omega+i \epsilon} \\
& +\frac{i}{2 \pi \sqrt{\omega \omega^{\prime}}} \int_{-\infty}^{v_{H}} d u\left[\partial_{u} \psi_{\omega \ell}^{\mathrm{in}}\left(u, v_{0}\right)\right] \psi_{\omega^{\prime} \ell}^{H^{+} *}\left(u_{s}(u), v_{0}\right) \\
& B_{\omega \omega^{\prime} l}^{H^{+}}=\frac{i}{2 \pi} \sqrt{\frac{\omega^{\prime}}{\omega}} \frac{e^{-i \omega^{\prime} v_{0}}}{\omega^{\prime}+i \epsilon} \psi_{\omega \ell}^{\text {in }}\left(v_{H}, v_{0}\right)-\frac{i}{2 \pi} \sqrt{\frac{\omega^{\prime}}{\omega}} \frac{1}{F_{L}\left(\omega^{\prime}, \ell\right)} \frac{e^{-i\left(\omega+\omega^{\prime}\right) v_{0}}}{\omega^{\prime}+\omega-i \epsilon} \\
& -\frac{i}{2 \pi \sqrt{\omega \omega^{\prime}}} \int_{-\infty}^{v_{H}} d u\left[\partial_{u} \psi_{\omega \ell}^{\mathrm{in}}\left(u, v_{0}\right)\right] \psi_{\omega^{\prime} \ell}^{H^{+}}\left(u_{s}(u), v_{0}\right) \text {, } \\
& A_{\omega \omega^{\prime} l}^{\mathcal{I}^{+}}=-\frac{i}{2 \pi} \sqrt{\frac{\omega^{\prime}}{\omega}} \frac{F_{R}^{*}\left(\omega^{\prime}, \ell\right)}{F_{L}^{*}\left(\omega^{\prime}, \ell\right)} \frac{e^{-i\left(\omega-\omega^{\prime}\right) v_{0}}}{\omega^{\prime}-\omega+i \epsilon}-\frac{i}{2 \pi \sqrt{\omega \omega^{\prime}}} \int_{-\infty}^{v_{H}} d u\left[\psi_{\omega \ell}^{\text {in }}\left(u, v_{0}\right)-e^{-i \omega v_{0}}\right] \partial_{u} \psi_{\omega^{\prime} \ell}^{\mathcal{I}^{+}}\left(u_{s}(u), v_{0}\right), \\
& B_{\omega \omega^{\prime} l}^{\mathcal{I}^{+}}=\frac{i}{2 \pi} \sqrt{\frac{\omega^{\prime}}{\omega}} \frac{F_{R}\left(\omega^{\prime}, \ell\right)}{F_{L}\left(\omega^{\prime}, \ell\right)} \frac{e^{-i\left(\omega+\omega^{\prime}\right) v_{0}}}{\omega^{\prime}+\omega-i \epsilon}+\frac{i}{2 \pi \sqrt{\omega \omega^{\prime}}} \int_{-\infty}^{v_{H}} d u\left[\psi_{\omega \ell}^{\mathrm{in}}\left(u, v_{0}\right)-e^{-i \omega v_{0}}\right] \partial_{u} \psi_{\omega^{\prime} \ell}^{\mathcal{I}^{+}}\left(u_{s}(u), v_{0}\right)
\end{aligned}
$$

\section{B. Expansions of modes for the Unruh state}

As discussed in Sec. III, our method for renormalizing the stress-energy tensor involves subtracting the unrenormalized stress-energy tensor for the Unruh modes. Recall these modes include the set of modes $f_{\omega \ell m}^{K}$ that are positive frequency on the past horizon with respect to the Kruskal time coordinate along with the set of modes $f_{\omega \ell m}^{\mathcal{I}^{-}}$that on $\mathcal{I}^{-}$have $\psi_{\omega \ell}=e^{-i \omega v}$. Before subtracting the contribution from the $f_{\omega \ell m}^{\mathcal{I}^{-}}$modes we first write them in terms of $f_{\omega \ell m}^{\mathcal{I}^{+}}$ and $f_{\omega \ell m}^{H^{+}}$using (4.13b).

For the contributions of the $f_{\omega \ell m}^{K}$ modes we first write down the Bogolubov transformation

$$
f_{\omega \ell m}^{K}=\int_{0}^{\infty} d \omega^{\prime}\left[\alpha_{\omega \omega^{\prime} \ell}^{K} f_{\omega^{\prime} \ell m}^{H^{-}}+\beta_{\omega \omega^{\prime} \ell}^{K} f_{\omega^{\prime} \ell m}^{H^{-} *}\right] .
$$

The coefficients can be obtained using the scalar product (2.15) with a Cauchy surface consisting of the union of past null infinity and the past horizon in Schwarzschild spacetime. Integrating over the angular coordinates one finds that the Bogolubov coefficients can be written in the form (5.5) with $\alpha$ replacing $A$ and $\beta$ replacing $B$. Integrating the remaining integrals over $u_{s}$ by parts one finds that ${ }^{3}$

$$
\begin{aligned}
\alpha_{\omega_{K} \omega^{\prime} \ell}^{K}= & \frac{1}{2 \pi} \sqrt{\frac{\omega^{\prime}}{\omega_{K}}}(4 M)^{1+i 4 M \omega^{\prime}} \\
& \times \int_{-\infty}^{0} d U_{K} e^{-i \omega_{K} U_{K}\left(-U_{K}\right)^{-1-i 4 M \omega^{\prime}}} \\
= & \frac{1}{2 \pi} \sqrt{\frac{\omega^{\prime}}{\omega_{K}}}(4 M)^{1+i 4 M \omega^{\prime}} \frac{\Gamma(\delta-i 4 M \omega)}{\left(-i \omega_{K}+\epsilon\right)^{-i 4 M \omega^{\prime}}}, \\
\beta_{\omega_{K}^{K} \omega^{\prime} \ell}= & \frac{1}{2 \pi} \sqrt{\frac{\omega^{\prime}}{\omega_{K}}}(4 M)^{1-i 4 M \omega^{\prime}} \\
& \times \int_{-\infty}^{0} d U_{K} e^{-i \omega_{K} U_{K}}\left(-U_{K}\right)^{-1+i 4 M \omega^{\prime}} \\
= & \frac{1}{2 \pi} \sqrt{\frac{\omega^{\prime}}{\omega_{K}}}(4 M)^{1-i 4 M \omega^{\prime}} \frac{\Gamma(\delta+i 4 M \omega)}{\left(-i \omega_{K}+\epsilon\right)^{i 4 M \omega^{\prime}}} .
\end{aligned}
$$

\footnotetext{
${ }^{3}$ This calculation was originally done in [32] but note that there is a mistake in the results. The expressions in that paper are missing a factor of $(4 M)^{ \pm i \omega^{\prime}}$.
} 
Here $\delta$ and $\epsilon$ are integrating factors with $0<\delta \ll 1$ and $0<\epsilon \ll 1$. Note that the Bogolubov coefficients are independent of the value of $\ell$. This is because the effective potential vanishes on $\mathrm{H}^{-}$which is the surface where the integrals are being computed. Then we use (4.13) to express the modes $f_{\omega \ell m}^{H^{-}, \mathcal{I}^{-}}$in terms of the modes $f_{\omega \ell m}^{\left(\mathcal{I}^{+} H^{+}\right)}$.

\section{2D example}

In this section we will illustrate the matching for the case of a 2D spacetime which has a perfectly reflecting mirror at $r=0$. The metric inside the shell is the flat space metric

$$
d s^{2}=-d t^{2}+d r^{2},
$$

and the metric outside the shell is the Schwarzschild metric

$$
d s^{2}=-\left(1-\frac{2 M}{r}\right) d t_{s}^{2}+\left(1-\frac{2 M}{r}\right)^{-1} d r^{2}
$$

The Penrose diagram is the same as in the $4 \mathrm{D}$ case as is the definition of the radial null coordinates $u, u_{s}$, and $v$ and the relation between $u$ and $u_{s}$.

The general form of the mode functions is

$$
f_{\omega}=\frac{\psi_{\omega}}{\sqrt{4 \pi \omega}} .
$$

There is no scattering for the massless minimally coupled scalar field modes in $2 \mathrm{D}$ so

$$
E_{R}=F_{L}=1, \quad E_{L}=F_{R}=0 .
$$

Inside the shell the in modes are

$$
\psi_{\omega}^{\text {in }}=e^{-i \omega v}-e^{-i \omega u} .
$$

In the region outside the shell the spacetime is the 2D version of Schwarzschild spacetime and the modes are

$$
\begin{aligned}
& \psi_{\omega}^{\mathcal{I}^{+}}=\psi_{\omega}^{H^{-}}=e^{-i \omega u_{s}}, \\
& \psi_{\omega}^{H^{+}}=\psi_{\omega}^{\mathcal{I}^{-}}=e^{-i \omega v} .
\end{aligned}
$$

The expansion for the in modes is similar to the 4D case except there are no parameters $\ell$ and $m$ related to the spherical harmonics. Thus

$$
\begin{aligned}
f_{\omega}^{\text {in }}= & \int_{0}^{\infty} d \omega^{\prime}\left[A_{\omega \omega^{\prime}}^{H^{+}} f_{\omega^{\prime}}^{H^{+}}+B_{\omega \omega^{\prime}}^{H^{+}}\left(f_{\omega^{\prime}}^{H^{+}}\right)^{*}+A_{\omega \omega^{\prime}}^{\mathcal{I}^{+}} f_{\omega^{\prime}}^{\mathcal{I}^{+}}\right. \\
& \left.+B_{\omega \omega^{\prime}}^{\mathcal{I}^{+}}\left(f_{\omega^{\prime}}^{\mathcal{I}^{+}}\right)^{*}\right] .
\end{aligned}
$$

The matching coefficients are given by substituting (5.21), (5.22), and (5.23) into (5.15). It is then easy to show that

$$
\begin{aligned}
& {\left[B_{\omega, \omega^{\prime}}^{H^{+}} f_{\omega^{\prime}}^{H^{+} *}\right]_{\omega^{\prime} \rightarrow-\omega^{\prime}}=A_{\omega, \omega^{\prime}}^{H^{+}} f_{\omega^{\prime}}^{H^{+}},} \\
& {\left[B_{\omega, \omega^{\prime}}^{\mathcal{I}^{+}} f_{\omega^{\prime}}^{\mathcal{I}^{+} *}\right]_{\omega^{\prime} \rightarrow-\omega^{\prime}}=A_{\omega, \omega^{\prime}}^{\mathcal{I}^{+}} f_{\omega^{\prime}}^{\mathcal{I}^{+}},}
\end{aligned}
$$

where the quantities on the right-hand side are to be evaluated at $\omega^{\prime}<0$. As a result

$$
f_{\omega}^{\text {in }}=\int_{-\infty}^{\infty} d \omega^{\prime}\left[A_{\omega \omega^{\prime}}^{H^{+}} f_{\omega^{\prime}}^{H^{+}}+A_{\omega \omega^{\prime}}^{\mathcal{I}^{+}} f_{\omega^{\prime}}^{\mathcal{I}^{+}}\right]
$$

Because $\psi_{\omega^{\prime}}^{H^{+}}$does not depend on $u$, the integral in (5.15a) is trivial to evaluate and one finds that

$$
A_{\omega \omega^{\prime}}^{H^{+}}=-\frac{i}{2 \pi} \sqrt{\frac{\omega^{\prime}}{\omega}} \frac{e^{-i\left(\omega-\omega^{\prime}\right) v_{0}}}{\omega^{\prime}-i \epsilon}+\frac{i}{2 \pi} \sqrt{\frac{\omega^{\prime}}{\omega}} \frac{e^{i\left(\omega^{\prime}-\omega\right) v_{0}}}{\omega^{\prime}-\omega+i \epsilon} .
$$

To see what the contribution to $f_{\omega}^{\text {in }}$ is from the $f_{\omega}^{H^{+}}$ modes, first substitute (5.27) into the first two terms of (5.24) along with (5.20) and (5.22) with the result

$$
\begin{aligned}
\left(f_{\omega}^{\mathrm{in}}\right)_{H^{+}} & =\frac{i e^{-i \omega v_{0}}}{2 \pi \sqrt{4 \pi \omega}} \int_{-\infty}^{\infty} d \omega^{\prime}\left[e^{i \omega^{\prime}\left(v_{0}-v\right)}\left(-\frac{1}{\omega^{\prime}-i \epsilon}+\frac{1}{\omega^{\prime}-\omega+i \epsilon}\right)\right] \\
& =\frac{e^{-i \omega v_{0}}}{\sqrt{4 \pi \omega}} \theta\left(v_{0}-v\right)+\frac{e^{-i \omega v}}{\sqrt{4 \pi \omega}} \theta\left(v-v_{0}\right) .
\end{aligned}
$$

We next consider the contribution of the $f^{\mathcal{I}^{+}}$modes. The matching coefficient in (5.15c) is

$$
\begin{aligned}
A_{\omega \omega^{\prime}}^{\mathcal{I}^{+}} & =-\frac{1}{2 \pi} \sqrt{\frac{\omega^{\prime}}{\omega}} \int_{-\infty}^{v_{H}} d u e^{-i \omega u} e^{i \omega^{\prime} u_{s}(u)} \frac{d u_{s}}{d u} \\
& =-\frac{1}{2 \pi} \sqrt{\frac{\omega^{\prime}}{\omega}} \int_{-\infty}^{v_{H}} d u e^{-i\left(\omega-\omega^{\prime}\right) u}\left(\frac{v_{H}-u}{4 M}\right)^{-i 4 M \omega^{\prime}}\left[1+\frac{4 M}{v_{h}-u}\right] .
\end{aligned}
$$

Changing variables to $x=v_{H}-u$ and performing an integration by parts gives 


$$
\begin{aligned}
A_{\omega \omega^{\prime}}^{\mathcal{I}^{+}} & =\frac{i}{2 \pi} \sqrt{\omega \omega^{\prime}} e^{-i\left(\omega-\omega^{\prime}\right) v_{H}} \frac{(4 M)^{1+i 4 M \omega^{\prime}}}{i\left(\omega^{\prime}-\omega\right)+\epsilon} \int_{0}^{\infty} d x e^{i\left(\omega-\omega^{\prime}\right) x-\epsilon x} x^{-i 4 M \omega^{\prime}-1+\delta} \\
& =\frac{i}{2 \pi} \sqrt{\omega \omega^{\prime}} e^{-i\left(\omega-\omega^{\prime}\right) v_{H}}(4 M)^{1+i 4 M \omega^{\prime}} \frac{\Gamma\left(\delta-i 4 M \omega^{\prime}\right)}{\left[i\left(\omega^{\prime}-\omega\right)+\epsilon\right]^{1-i 4 M \omega^{\prime}}} .
\end{aligned}
$$

Note that two integrating factors have been used with $0<\epsilon \ll 1$ and $0<\delta \ll 1$.

To find the contribution to $f_{\omega}^{\text {in }}$ from the $f_{\omega}^{\mathcal{I}^{+}}$modes, first substitute (5.30) into (5.26) with the result

$$
\left(f_{\omega}^{\mathrm{in}}\right)_{\mathcal{I}^{+}}=\frac{i 4 M \sqrt{\omega}}{2 \pi \sqrt{4 \pi}} e^{-i \omega v_{H}} \int_{-\infty}^{\infty} d \omega^{\prime} e^{i \omega^{\prime}\left(v_{H}-u_{s}\right)}(4 M)^{i 4 M \omega^{\prime}} \frac{\Gamma\left(\delta-i 4 M \omega^{\prime}\right)}{\left[i\left(\omega^{\prime}-\omega\right)+\epsilon\right]^{1-i 4 M \omega^{\prime}}} .
$$

Note that the denominator has an essential singularity in the upper half $\omega^{\prime}$ plane while the Gamma function has simple poles in the lower half plane at

$$
\omega^{\prime}=-\frac{i \delta}{4 M}
$$

and

$$
\omega^{\prime}=-\frac{\text { in }}{4 M}, \quad n=1,2, \ldots
$$

In the complex plane at large $\left|\omega^{\prime}\right|$ Sterling's approximation gives

$\Gamma\left(-i 4 M \omega^{\prime}\right) \approx \sqrt{2 \pi} e^{i 4 M \omega^{\prime}} e^{\left(-i 4 M \omega^{\prime}-1 / 2\right) \log \left(-i 4 M \omega^{\prime}\right)}$.

Using the usual change of variables $\omega^{\prime}=R e^{i \theta}$, with $R>0$, it is straightforward to show that the dominant contribution to the integrand of (5.31) in the large $R$ limit comes from the factor $e^{4 M R \sin \theta \log R}$ and therefore one must close in the lower half plane. This means there is no contribution from the essential singularity but there is a contribution from each pole of the Gamma function. At these poles it is straightforward to show that

$\Gamma(\delta-i 4 M \omega) \rightarrow \frac{(-1)^{n}}{n !(n-i 4 M \omega)}, \quad n=0,1,2, \ldots$

Then

$$
\begin{aligned}
\left(f_{\omega}^{\mathrm{in}}\right)_{\mathcal{I}^{+}}= & \frac{4 M i \sqrt{\omega}}{\sqrt{4 \pi}} e^{-i \omega v_{H}} \sum_{n=0}^{\infty} \frac{(-1)^{n}}{n !}(n-i 4 M \omega)^{n-1} \\
& \times\left[\exp \left(\frac{\left(v_{H}-u_{S}\right)}{4 M}\right)\right]^{n} .
\end{aligned}
$$

Because the general solutions to the 2D mode equation in Schwarzschild spacetime are of the form $\psi=g\left(u_{s}\right)+h(v)$ with $g$ and $h$ arbitrary functions, the exact solution for the in modes is

$$
\begin{aligned}
\left(f_{\omega}^{\text {in }}\right)_{\mathcal{I}^{+}} & =-\frac{e^{-i \omega u\left(u_{s}\right)}}{\sqrt{4 \pi \omega}} \\
& =-\frac{e^{-i \omega v_{H}}}{\sqrt{4 \pi \omega}} \exp \left\{i 4 M \omega W\left[\exp \left(\frac{\left(v_{H}-u_{s}\right)}{4 M}\right)\right]\right\},
\end{aligned}
$$

where (2.8) has been used and $W(z)$ is the Lambert $\mathrm{W}$ function. To make a comparison between (5.36) and (5.37), one needs to write the latter in terms of a series. This has been done in [33]. An alternative derivation is given in Appendix A. The result is

$$
e^{-c W(z)}=\sum_{n=0}^{\infty} \frac{c(n+c)^{n-1}}{(n) !}(-z)^{n} .
$$

Taking $c=-4 i M \omega$ and $z=\exp \left(\frac{v_{H}-u_{s}}{4 M}\right)$ in (5.38), one can see that (5.37) and (5.36) are equivalent.

\section{Delta function potential}

In this section, we apply our matching method to the case where the potential term in (4.2) is replaced by

$$
V=\lambda \delta\left(r_{*}\right)
$$

with $\lambda$ a positive real constant. This can serve as a model for the original potential which has a single peak and vanishes at the horizon and infinity. The resulting mode equation can be solved analytically and the solutions are simple enough that the matching coefficients can be computed analytically. Some of these matching coefficients will be used to partially reconstruct the mode functions $f_{\omega \ell}^{\text {in }}$ in the case that $\ell=0$.

For $\ell=0$ in 4D the in modes inside the null shell take on the particularly simple form (2.21). In the region outside the shell the mode functions in the complete set with $\ell=0$ have the general form

$$
f_{\omega^{\prime} 00}^{\left(H^{+}, \mathcal{I}^{+}\right)}=\frac{Y_{00}}{r \sqrt{4 \pi \omega^{\prime}}} \psi_{\omega^{\prime} 0}^{\left(H^{+}, \mathcal{I}^{+}\right)}, \quad \psi_{\omega^{\prime} 0}^{\left(H^{+}, \mathcal{I}^{+}\right)}=e^{-i \omega^{\prime} t_{s}} \chi_{\omega^{\prime} 0}^{\left(H^{+}, \mathcal{I}^{+}\right)} .
$$


The radial parts of the modes satisfy the following equation:

$$
\frac{d^{2} \chi}{d r_{*}^{2}}+\left(\omega^{2}-\lambda \delta\left(r_{*}\right)\right) \chi=0
$$

In the region where $r_{*}>0$, two linearly independent solutions are

$$
\begin{gathered}
\chi_{R}^{\infty}=e^{i \omega r_{*}}, \\
\chi_{L}^{\infty}=e^{-i \omega r_{*}} .
\end{gathered}
$$

For $r_{*}<0, \chi_{R}$ and $\chi_{L}$ can be expressed in the following way:

$$
\begin{aligned}
& \chi_{R}^{\infty}=E_{R} e^{i \omega r_{*}}+F_{R} e^{-i \omega r_{*}}, \\
& \chi_{L}^{\infty}=E_{L} e^{i \omega r_{*}}+F_{L} e^{-i \omega r_{*}} .
\end{aligned}
$$

Imposing the continuity of the mode function and discontinuity of its first derivative in the usual way at the spacelike curve $r_{*}=0$, the following analytic expressions are found for the scattering coefficients

$$
\begin{gathered}
E_{R}=1+\frac{i \lambda}{2 \omega}, \\
F_{R}=-\frac{i \lambda}{2 \omega}, \\
E_{L}=F_{R}^{*}=\frac{i \lambda}{2 \omega}, \\
F_{L}=E_{R}^{*}=1-\frac{i \lambda}{2 \omega} .
\end{gathered}
$$

Then the mode functions that we are using for the matching can be obtained from (4.5) and (4.7) with the result

$$
\begin{aligned}
& \psi_{\omega^{\prime} 0}^{H^{+}}=\theta\left(-r_{*}\right)\left[e^{-i \omega^{\prime} v}+\frac{\frac{i \lambda}{2}}{\left(\omega^{\prime}-\frac{i \lambda}{2}\right)} e^{-i \omega^{\prime} u_{s}}\right]+\theta\left(r_{*}\right) \frac{\omega^{\prime}}{\left(\omega^{\prime}-\frac{i \lambda}{2}\right)} e^{-i \omega^{\prime} v}, \\
& \psi_{\omega^{\prime} 0}^{\mathcal{I}^{+}}=\theta\left(-r_{*}\right) \frac{\omega^{\prime}}{\left(\omega^{\prime}-\frac{i \lambda}{2}\right)} e^{-i \omega^{\prime} u_{s}}+\theta\left(r_{*}\right)\left[\frac{\frac{i \lambda}{2}}{\left(\omega^{\prime}-\frac{i \lambda}{2}\right)} e^{-i \omega^{\prime} v}+e^{-i \omega^{\prime} u_{s}}\right] .
\end{aligned}
$$

To verify that the matching coefficients can be used to reconstruct the original mode functions for the case $\ell=0$ it is useful to break them up into contributions that come from the term proportional to $e^{-i \omega v}$ in (2.21) and the term proportional to $-e^{-i \omega u}$. In what follows we compute the matching coefficients for both terms but then focus only on those that come from the term proportional to $e^{-i \omega v}$. Substituting (5.45), and (5.44) into (5.15) one finds the matching coefficients

$$
\begin{aligned}
& A_{\omega \omega^{\prime} 0}^{H^{+}}=\left(A_{\omega \omega^{\prime} 0}^{H^{+}}\right)_{v}+\left(A_{\omega \omega^{\prime} 0}^{H^{+}}\right)_{u}, \\
& \left(A_{\omega \omega^{\prime} 0}^{H^{+}}\right)_{v}=-\frac{i}{2 \pi} \sqrt{\frac{\omega^{\prime}}{\omega}} \frac{e^{i \omega^{\prime} v_{0}}}{\omega^{\prime}-i \epsilon} e^{-i \omega v_{0}}+\frac{i}{2 \pi} \sqrt{\frac{\omega^{\prime}}{\omega}} \frac{\omega^{\prime}}{\left(\omega^{\prime}+\frac{i \lambda}{2}\right)} \frac{e^{i\left(\omega^{\prime}-\omega\right) v_{0}}}{\left(\omega^{\prime}-\omega+i \epsilon\right)}, \\
& \left(A_{\omega \omega^{\prime} 0}^{H^{+}}\right)_{u}=\frac{i}{2 \pi} \sqrt{\frac{\omega^{\prime}}{\omega}} \frac{e^{i \omega^{\prime} v_{0}}}{\omega^{\prime}-i \epsilon} e^{-i \omega v_{H}}-\frac{1}{2 \pi} \sqrt{\frac{\omega}{\omega^{\prime}}} \int_{-\infty}^{v_{H}} d u e^{-i \omega u}\left[\theta\left(r_{*}\right) \frac{\omega^{\prime}}{\omega^{\prime}+\frac{i \lambda}{2}} e^{i \omega^{\prime} v_{0}}\right. \\
& \left.+\theta\left(-r_{*}\right)\left(e^{i \omega^{\prime} v_{0}}-\frac{\frac{i \lambda}{2}}{\omega^{\prime}+\frac{i \lambda}{2}} e^{i \omega^{\prime} u_{s}(u)}\right)\right], \\
& A_{\omega \omega^{\prime} 0}^{\mathcal{I}^{+}}=\left(A_{\omega \omega^{\prime} 0}^{\mathcal{I}^{+}}\right)_{v}+\left(A_{\omega \omega^{\prime} 0}^{\mathcal{I}^{+}}\right)_{u}, \\
& \left(A_{\omega \omega^{\prime} 0}^{\mathcal{I}^{+}}\right)_{v}=-\frac{i}{2 \pi} \sqrt{\frac{\omega^{\prime}}{\omega}} \frac{\frac{i \lambda}{2}}{\left(\omega^{\prime}+\frac{i \lambda}{2}\right)} \frac{e^{-i\left(\omega-\omega^{\prime}\right) v_{0}}}{\left(\omega^{\prime}-\omega+i \epsilon\right)}, \\
& \left(A_{\omega \omega^{\prime} 0}^{\mathcal{I}^{+}}\right)_{u}=-\frac{1}{2 \pi} \sqrt{\frac{\omega^{\prime}}{\omega}} \int_{-\infty}^{v_{H}} d u \frac{d u_{s}(u)}{d u} e^{-i \omega u} e^{i \omega^{\prime} u_{s}(u)}\left[\theta\left(r_{*}\right)+\theta\left(-r_{*}\right) \frac{\omega^{\prime}}{\omega^{\prime}+\frac{i \lambda}{2}}\right] .
\end{aligned}
$$

Note that the relations (5.25) are satisfied by these matching coefficients so the relation (5.26) also holds. Thus 


$$
\left(f_{\omega 00}^{\mathrm{in}}\right)_{v}=\int_{-\infty}^{\infty} d \omega^{\prime}\left[\left(A_{\omega \omega^{\prime} 0}^{H^{+}}\right)_{v} f_{\omega^{\prime} 00}^{H^{+}}+\left(A_{\omega \omega^{\prime} 0}^{\mathcal{I}^{+}}\right)_{v} f_{\omega^{\prime} 00}^{\mathcal{I}^{+}}\right]
$$

Substituting (5.46a), (5.47a), and (5.45) into (5.48) gives after some algebra

$$
\begin{aligned}
\left(f_{\omega 00}^{\text {in }}\right)_{v}= & \frac{Y_{00}}{r \sqrt{4 \pi \omega}}\left[\theta\left(-r_{*}\right) I_{1}+\theta\left(r_{*}\right) I_{2}\right] \\
I_{1}= & -\frac{i}{2 \pi} e^{-i \omega v_{0}} \int_{-\infty}^{\infty} d \omega^{\prime}\left[\frac{\frac{i \lambda}{2} e^{i \omega^{\prime}\left(v_{0}-u_{s}\right)}}{\left(\omega^{\prime}-i \epsilon\right)\left(\omega^{\prime}-\frac{i \lambda}{2}\right)}+\frac{e^{i \omega^{\prime}\left(v_{0}-v\right)}}{\left(\omega^{\prime}-i \epsilon\right)}-\frac{\omega^{\prime} e^{i \omega^{\prime}\left(v_{0}-v\right)}}{\left(\omega^{\prime}+\frac{i \lambda}{2}\right)\left(\omega^{\prime}-\omega+i \epsilon\right)}\right] \\
= & \theta\left(v_{0}-u_{s}\right) e^{-i \omega v_{0}}\left[e^{-\frac{\lambda}{2}\left(v_{0}-u_{s}\right)}-1\right]+\theta\left(v_{0}-v\right) e^{-i \omega v_{0}} \\
& +\frac{\theta\left(v-v_{0}\right)}{\left(\omega+\frac{i \lambda}{2}\right)}\left[\frac{i \lambda}{2} e^{-i \omega v_{0}} e^{-\frac{\lambda}{2}\left(v-v_{0}\right)}+\omega e^{-i \omega v}\right], \\
I_{2}= & -\frac{i}{2 \pi} e^{-i \omega v_{0}} \int_{-\infty}^{\infty} d \omega^{\prime}\left[\frac{\omega^{\prime} e^{i \omega^{\prime}\left(v_{0}-v\right)}}{\left(\omega^{\prime}-i \epsilon\right)\left(\omega^{\prime}-\frac{i \lambda}{2}\right)}-\frac{e^{i \omega^{\prime}\left(v_{0}-v\right)}}{\omega^{\prime}-\omega+i \epsilon}+\frac{\frac{i \lambda}{2} e^{i \omega^{\prime}\left(v_{0}-u_{s}\right)}}{\left(\omega^{\prime}-\omega+i \epsilon\right)\left(\omega^{\prime}+\frac{i \lambda}{2}\right)}\right] \\
= & \theta\left(v_{0}-v\right) e^{-i \omega v_{0}} e^{-\frac{\lambda}{2}\left(v_{0}-v\right)}+\theta\left(v-v_{0}\right) e^{-i \omega v} \\
& -\theta\left(u_{s}-v_{0}\right) \frac{\frac{i \lambda}{2}}{\left(\omega+\frac{i \lambda}{2}\right)}\left[-e^{-i \omega v_{0}} e^{-\frac{\lambda}{2}\left(u_{s}-v_{0}\right)}+e^{-i \omega u_{s}}\right] .
\end{aligned}
$$

It is easy to verify that (5.49) gives the correct values for $\left(f_{\omega 00}^{\text {in }}\right)_{v}$ on the future horizon for $v \leq v_{0}$, on the null shell surface $v=v_{0}$, and on past null infinity for $v \geq v_{0}$.

\section{E. Partial analytic results for the matching coefficients in 4D for $\mathscr{\ell}=0$}

Because of the simple form of the in modes for $\ell=0$ inside the null shell (2.21), it is possible to compute the matching coefficients for the $e^{-i \omega v}$ part analytically. To do so we begin by substituting (2.21) into (5.15) with the result

$$
\begin{gathered}
A_{\omega \omega^{\prime} 0}^{H^{+}}=-\frac{i}{2 \pi} \sqrt{\frac{\omega^{\prime}}{\omega}} \frac{e^{i \omega^{\prime} v_{0}}}{\omega^{\prime}-i \epsilon}\left(e^{-i \omega v_{0}}-e^{-i \omega v_{H}}\right)+\frac{i}{2 \pi} \sqrt{\frac{\omega^{\prime}}{\omega}} \frac{1}{F_{L}^{*}\left(\omega^{\prime}, 0\right)} \frac{e^{i\left(\omega^{\prime}-\omega\right) v_{0}}}{\omega^{\prime}-\omega+i \epsilon} \\
-\frac{1}{2 \pi} \sqrt{\frac{\omega}{\omega^{\prime}}} \int_{-\infty}^{v_{H}} d u e^{-i \omega u} \psi_{\omega^{\prime} 0}^{H^{+} *}\left(u_{s}(u), v_{0}\right), \\
B_{\omega \omega^{\prime} 0}^{H^{+}}=\frac{i}{2 \pi} \sqrt{\frac{\omega^{\prime}}{\omega}} \frac{e^{-i \omega^{\prime} v_{0}}}{\omega^{\prime}+i \epsilon}\left(e^{-i \omega v_{0}}-e^{-i \omega v_{H}}\right)-\frac{i}{2 \pi} \sqrt{\frac{\omega^{\prime}}{\omega}} \frac{1}{F_{L}\left(\omega^{\prime}, 0\right)} \frac{e^{-i\left(\omega+\omega^{\prime}\right) v_{0}}}{\omega^{\prime}+\omega-i \epsilon} \\
+\frac{1}{2 \pi} \sqrt{\frac{\omega}{\omega^{\prime}}} \int_{-\infty}^{v_{H}} d u e^{-i \omega u} \psi_{\omega^{\prime} 0}^{H^{+}}\left(u_{s}(u), v_{0}\right), \\
A_{\omega \omega^{\prime} 0}^{\mathcal{I}^{+}}=-\frac{i}{2 \pi} \sqrt{\frac{\omega^{\prime}}{\omega}} \frac{F_{R}^{*}\left(\omega^{\prime}, 0\right)}{F_{L}^{*}\left(\omega^{\prime}, 0\right)} \frac{e^{-i\left(\omega-\omega^{\prime}\right) v_{0}}}{\omega^{\prime}-\omega+i \epsilon}+\frac{i}{2 \pi} \frac{1}{\sqrt{\omega \omega^{\prime}}} \int_{-\infty}^{v_{H}} d u e^{-i \omega u} \partial_{u} \psi_{\omega^{\prime} 0}^{\mathcal{I}^{+} *}\left(u_{s}(u), v_{0}\right), \\
B_{\omega \omega^{\prime} 0}^{\mathcal{I}^{+}}=\frac{i}{2 \pi} \sqrt{\frac{\omega^{\prime}}{\omega}} \frac{F_{R}\left(\omega^{\prime}, 0\right)}{F_{L}\left(\omega^{\prime}, 0\right)} \frac{e^{-i\left(\omega+\omega^{\prime}\right) v_{0}}}{\omega^{\prime}+\omega-i \epsilon}-\frac{i}{2 \pi} \frac{1}{\sqrt{\omega \omega^{\prime}}} \int_{-\infty}^{v_{H}} d u e^{-i \omega u} \partial_{u} \psi_{\omega^{\prime} 0}^{\mathcal{I}^{+}}\left(u_{s}(u), v_{0}\right) .
\end{gathered}
$$

Note that the integrals have to be computed numerically because the mode functions in Schwarzschild spacetime must be computed numerically. However, because of the simple form that $\psi_{\omega 0}^{\mathrm{in}}$ takes it is possible to separate the matching coefficients into separate matching coefficients for the part that goes like $e^{-i \omega v}$ inside the null shell and the part that goes like $e^{-i \omega u}$ there. The matching coefficients for $e^{-i \omega v}$ do not depend on the integrals. In what follows we focus on these matching coefficients. Examination of (5.50) gives for these coefficients 


$$
\begin{aligned}
& \left(A_{\omega \omega^{\prime} 0}^{H^{+}}\right)_{v}=-\frac{i}{2 \pi} \sqrt{\frac{\omega^{\prime}}{\omega}} \frac{e^{i\left(\omega^{\prime}-\omega\right) v_{0}}}{\omega^{\prime}-i \epsilon}+\frac{i}{2 \pi} \sqrt{\frac{\omega^{\prime}}{\omega}} \frac{1}{F_{L}^{*}\left(\omega^{\prime}, 0\right)} \frac{e^{i\left(\omega^{\prime}-\omega\right) v_{0}}}{\omega^{\prime}-\omega+i \epsilon}, \\
& \left(B_{\omega \omega^{\prime} l}^{H^{+}}\right)_{v}=\frac{i}{2 \pi} \sqrt{\frac{\omega^{\prime}}{\omega}} \frac{e^{-i\left(\omega^{\prime}+\omega\right) v_{0}}}{\omega^{\prime}+i \epsilon}-\frac{i}{2 \pi} \sqrt{\frac{\omega^{\prime}}{\omega}} \frac{1}{F_{L}\left(\omega^{\prime}, 0\right)} \frac{e^{-i\left(\omega+\omega^{\prime}\right) v_{0}}}{\omega^{\prime}+\omega-i \epsilon}, \\
& \left(A_{\omega \omega^{\prime} l}^{\mathcal{I}^{+}}\right)_{v}=-\frac{i}{2 \pi} \sqrt{\frac{\omega^{\prime}}{\omega}} \frac{F_{R}^{*}\left(\omega^{\prime}, 0\right)}{F_{L}^{*}\left(\omega^{\prime}, 0\right)} \frac{e^{-i\left(\omega-\omega^{\prime}\right) v_{0}}}{\omega^{\prime}-\omega+i \epsilon}, \\
& \left(B_{\omega^{\prime} \omega^{\prime} l}^{\mathcal{I}^{+}}\right)_{v}=\frac{i}{2 \pi} \sqrt{\frac{\omega^{\prime}}{\omega}} \frac{F_{R}\left(\omega^{\prime}, 0\right)}{F_{L}\left(\omega^{\prime}, 0\right)} \frac{e^{-i\left(\omega+\omega^{\prime}\right) v_{0}}}{\omega^{\prime}+\omega-i \epsilon} .
\end{aligned}
$$

These matching coefficients can be used to reconstruct the part of the mode function which goes like $e^{-i \omega v}$ inside the shell by substituting the expressions into (5.1). To check them we shall compute the resulting integral on $\mathrm{H}^{+}$. Recall that we are working in the exact Schwarzschild spacetime rather than the null shell spacetime when we do the matching. The same applies to the reconstruction. Thus the results for the reconstruction for which $v \geq v_{0}$ also apply to the null shell spacetime, but the results for $v<v_{0}$ do not apply to the null shell spacetime.

Recall that the modes $f^{\mathcal{I}^{+}}$vanish on $H^{+}$.

$$
\begin{aligned}
\left(f_{\omega 00}^{\mathrm{in}}\right)_{v} & =\frac{Y_{00}}{r \sqrt{4 \pi \omega}}\left(I_{1}+I_{2}\right), \\
I_{1} & =-\frac{i}{2 \pi} e^{-i \omega v_{0}} \int_{0}^{\infty} d \omega^{\prime}\left[\frac{e^{i\left(v_{0}-v\right) \omega^{\prime}}}{\omega^{\prime}-i \epsilon}-\frac{e^{-i\left(v_{0}-v\right) \omega^{\prime}}}{\omega^{\prime}+i \epsilon}\right],
\end{aligned}
$$

$$
\begin{aligned}
I_{2}= & \frac{i}{2 \pi} e^{-i \omega v_{0}} \int_{0}^{\infty} d \omega^{\prime}\left[\frac{1}{F_{L}^{*}\left(\omega^{\prime}, 0\right)} \frac{e^{-i \omega^{\prime}\left(v-v_{0}\right)}}{\omega^{\prime}-\omega+i \epsilon}\right. \\
& \left.-\frac{1}{F_{L}\left(\omega^{\prime}, 0\right)} \frac{e^{i \omega^{\prime}\left(v-v_{0}\right)}}{\omega^{\prime}+\omega-i \epsilon}\right] .
\end{aligned}
$$

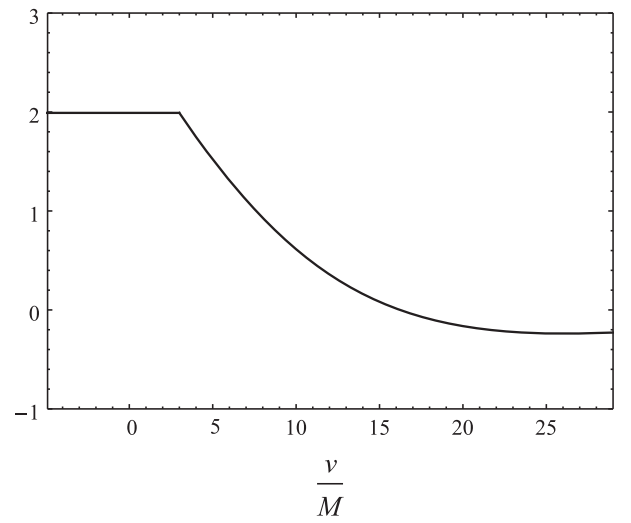

If in the second term of $I_{1}$ a change of variables is made so that $\omega^{\prime} \rightarrow-\omega^{\prime}$ then one finds that

$$
I_{1}=-\frac{i}{2 \pi} e^{-i \omega v_{0}} \int_{-\infty}^{\infty} d \omega^{\prime} \frac{e^{i\left(v_{0}-v\right) \omega^{\prime}}}{\omega^{\prime}-i \epsilon}=e^{-i \omega v_{0}} \theta\left(v_{0}-v\right)
$$

with $\theta$ the step function. It is thus clear that the initial data on $H^{+}$for $-\infty<v<v_{0}$ does not affect the mode functions on the part of the future horizon for which $v_{0}<v<\infty$.

It can be shown from the properties of the scattering coefficients given in [31], that $F_{L}\left(\omega^{\prime}\right)=F_{L}^{*}\left(-\omega^{\prime}\right)$. Using this identity and changing the variable of integration in the second integral in the same way as was done for $I_{1}$, one obtains

$$
\begin{aligned}
\left(f_{\omega 00}^{\mathrm{in}}\right)_{v}= & \frac{Y_{00} e^{-i \omega v_{0}}}{r \sqrt{4 \pi \omega}} \theta\left(v_{0}-v\right) \\
& +\frac{i}{2 \pi \sqrt{4 \pi \omega}} \int_{-\infty}^{\infty} d \omega^{\prime} \frac{e^{-i \omega^{\prime} v}}{F_{L}^{*}\left(\omega^{\prime}, 0\right)} \frac{e^{i v_{0}\left(\omega^{\prime}-\omega\right)}}{\omega^{\prime}-\omega+i \epsilon} .
\end{aligned}
$$

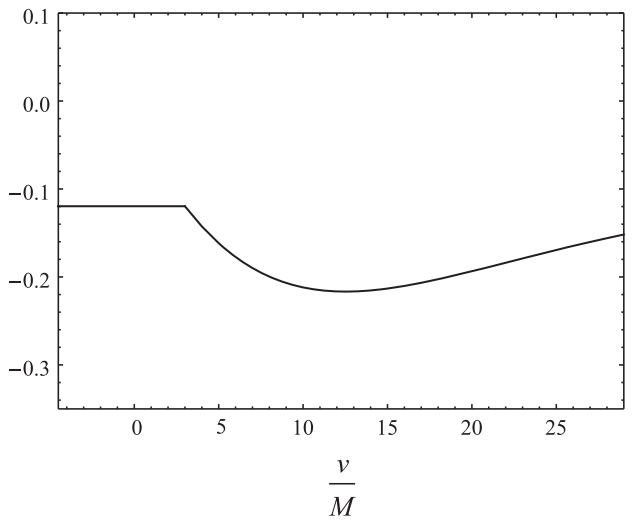

FIG. 3. The real (left) and the imaginary (right) parts of $\sqrt{\frac{4 \pi}{M}}\left(f_{\omega 00}^{\text {in }}\right)_{v}$ on the future horizon have been plotted. In both plots, $M \omega=0.02$ and $\frac{v_{0}}{M}=3$. The plots clearly show that $\left(f_{\omega 00}^{\text {in }}\right)_{v}$ is continuous at $v=v_{0}$. 
To compute this integral using complex integration techniques one must know the singularity structure of $\frac{1}{F_{L}^{*}}$ which is difficult since this scattering coefficient must be computed numerically. However, one can at least test whether it has one or more singularities in the complex plane by assuming it does not and computing the integral. We will call the result $f^{\text {test }}$ because there is no guarantee that this method will give the correct answer. The result of such an integration is

$$
f^{\mathrm{test}}=\frac{e^{-i \omega v_{0}}}{\sqrt{4 \pi \omega}} \theta\left(v_{0}-v\right)+\frac{e^{-i \omega v}}{F_{L}^{*}\left(\omega^{\prime}, 0\right) \sqrt{4 \pi \omega}} \theta\left(v-v_{0}\right) .
$$

Here complex integration has been performed using a contour in the lower half of the complex plane. It is obvious that at $v=v_{0}$, the continuity condition for $\left(f_{\omega 00}^{\text {in }}\right)_{v}$ is not satisfied so $f^{\text {test }} \neq\left(f_{\omega 00}^{\text {in }}\right)_{v}$, which implies that $\frac{1}{F_{L}^{*}\left(\omega^{\prime}\right)}$ has one or more singularities in the complex plane.

Alternatively one can work with $I_{2}$ in the form $(5.52 \mathrm{~b})$, use the relation $\left(\omega^{\prime} \mp \omega \pm i \epsilon\right)^{-1}=\mp i \pi \delta\left(\omega^{\prime} \mp \omega\right)+$ $\left(\omega^{\prime} \mp w\right)^{-1}$, and compute the principle value parts of the integral numerically for $v>v_{0}$. This has been done and the result is shown in Fig. 3. It is clear from the plots in this figure that on the future horizon $\left(f_{\omega 00}^{\mathrm{in}}\right)_{v}$ is continuous at $v=v_{0}$.

\section{STRESS-ENERGY TENSOR}

\section{A. Method in 4D}

For the massless minimally coupled scalar field the classical stress-energy tensor in a general curved spacetime is given in (3.1) and a renormalization expression for $\left\langle T_{a b}\right\rangle$ is given in (3.2). To compute $\left\langle T_{a b}\right\rangle$ using (3.2) it is useful to begin with the points split and to write the stress-energy tensor in terms of derivatives of the Hadamard Green's function

$$
G^{(1)}\left(x, x^{\prime}\right)=\left\langle\left\{\phi(x), \phi\left(x^{\prime}\right)\right\}\right\rangle .
$$

We adopt the notation

$\Delta G^{(1)}\left(x, x^{\prime}\right)=\left\langle\operatorname{in}\left|\left\{\phi(x), \phi\left(x^{\prime}\right)\right\}\right|\right.$ in $\rangle-\left\langle U\left|\left\{\phi(x), \phi\left(x^{\prime}\right)\right\}\right| U\right\rangle$,

with $\mid$ in $\rangle$ representing the in vacuum state and $|U\rangle$ the Unruh state. The corresponding difference in the stressenergy tensors is then

$$
\Delta\left\langle T_{a b}\right\rangle=\frac{1}{4} \lim _{x^{\prime} \rightarrow x}\left[\left(g_{a}^{c^{\prime}} \Delta G_{; c^{\prime} ; b}^{(1)}+g_{b}^{c^{\prime}} \Delta G_{; ; ; c^{\prime}}^{(1)}\right)-g_{a b} g^{c d^{\prime}} \Delta G_{; c ; d^{\prime}}^{(1)}\right] .
$$

Here the quantity $g_{a}^{b^{\prime}}$ parallel transports a vector from $x^{\prime}$ to $x$ and is called the bivector of parallel transport [34]. To leading order when the point separation is small

$$
g_{a}^{b^{\prime}}=g_{a}^{b}=\delta_{a}^{b} .
$$

The subleading orders all vanish in the limit $x^{\prime} \rightarrow x$. Since there are no ultraviolet divergences in the quantity $\Delta\left\langle T_{a b}\right\rangle$ one can use (6.4) in (6.3) with the result

$\Delta\left\langle T_{a b}\right\rangle=\frac{1}{4}\left[\lim _{x^{\prime} \rightarrow x}\left(\Delta G_{; a^{\prime} ; b}^{(1)}+\Delta G_{; a ; b^{\prime}}^{(1)}\right)-g_{a b} g^{c d} \lim _{x^{\prime} \rightarrow x} \Delta G_{; c ; d^{\prime}}^{(1)}\right]$,

where a slight abuse of notation has been used for the implied sum over $d$ and $d^{\prime}$ in the last term. It is important to note that this expression is valid in both two and four dimensions.

Expanding the field in terms of modes as in (2.10) one finds for the in modes that

$$
\left\langle 0 \text { in }\left|\left\{\phi(x), \phi\left(x^{\prime}\right)\right\}\right| 0 \text { in }\right\rangle=\sum_{\ell=0}^{\infty} \sum_{m=-\ell}^{\ell} \int_{0}^{\infty} d \omega\left[f_{\omega \ell m}^{\text {in }}(x)\left(f_{\omega \ell m}^{\text {in }}\left(x^{\prime}\right)\right)^{*}+f_{\omega \ell m}^{\text {in }}\left(x^{\prime}\right)\left(f_{\omega \ell m}^{\text {in }}(x)\right)^{*}\right] .
$$

The Unruh state in Schwarzschild spacetime consists of modes that are positive frequency with respect to the usual time coordinate on $\mathcal{I}^{-}$along with modes that are positive frequency with respect to the Kruskal time coordinate on $H^{-}$so that

$$
\begin{aligned}
\left\langle U\left|\left\{\phi(x), \phi\left(x^{\prime}\right)\right\}\right| U\right\rangle= & \sum_{\ell=0}^{\infty} \sum_{m=-\ell}^{\ell}\left\{\int_{0}^{\infty} d \omega_{K}\left[f_{\omega_{K} \ell m}^{K}(x)\left(f_{\omega_{K} \ell m}^{K}\left(x^{\prime}\right)\right)^{*}+f_{\omega_{K} \ell m}^{K}\left(x^{\prime}\right)\left(f_{\omega_{K} \ell m}^{K}(x)\right)^{*}\right]\right. \\
& \left.+\int_{0}^{\infty} d \omega\left[f_{\omega \ell m}^{\mathcal{I}^{-}}(x)\left(f_{\omega \ell m}^{\mathcal{I}^{-}}\left(x^{\prime}\right)\right)^{*}+f_{\omega \ell m}^{\mathcal{I}^{-}}\left(x^{\prime}\right)\left(f_{\omega \ell m}^{\mathcal{I}^{-}}(x)\right)^{*}\right]\right\} .
\end{aligned}
$$

The next step is to find expansions for these two-point functions in terms of the complete set of modes $f^{\left(\mathcal{I}^{+}, H^{+}\right)}$that we are using. For $\left\langle\operatorname{0in}\left|\left\{\phi(x), \phi\left(x^{\prime}\right)\right\}\right| \operatorname{0in}\right\rangle$ one can substitute (5.1) into (6.6). This results in integrals of the form 


$$
\begin{aligned}
& \sum_{\ell=0}^{\infty} \sum_{m=-\ell}^{\ell} \int_{0}^{\infty} d \omega \int_{0}^{\infty} d \omega_{1} \int_{0}^{\infty} d \omega_{2}\left\{\left[A^{\left(H^{+}, \mathcal{I}^{+}\right)} f^{\left(H^{+}, \mathcal{I}^{+}\right)}+B^{\left(H^{+}, \mathcal{I}^{+}\right)}\left(f^{\left(H^{+}, \mathcal{I}^{+}\right)}\right)^{*}\right]\right. \\
& \left.\quad \times\left[\left(A^{\left(H^{+}, \mathcal{I}^{+}\right)}\right) *\left(f^{\left(H^{+}, \mathcal{I}^{+}\right)}\right)^{*}+\left(B^{\left(H^{+}, \mathcal{I}^{+}\right)}\right)^{*} f^{\left(H^{+}, \mathcal{I}^{+}\right)}\right]\right\},
\end{aligned}
$$

where the subscripts on the matching coefficients and mode functions have been suppressed. For $\left\langle U\left|\left\{\phi(x), \phi\left(x^{\prime}\right)\right\}\right| U\right\rangle$ one can first substitute (5.16) and (5.17) into (6.7) to obtain an expression in terms of $f_{\omega \ell m}^{\left(H^{-}, \mathcal{I}^{-}\right)}$. Then (4.13) can be used to obtain an expression for $\left\langle U\left|\left\{\phi(x), \phi\left(x^{\prime}\right)\right\}\right| U\right\rangle$ that depends only on $f_{\omega \ell m}^{\left(\mathcal{I}^{+}, H^{+}\right)}$.

\section{B. 2D Example}

In this section the method discussed above is tested by using it to computing the stress-energy tensor for the scalar field in the corresponding 2D spacetime where the answer is known. The computation will be done in the region $v>v_{0}$ outside the null shell and outside the horizon. From (5.28) it is clear that for $v>v_{0}$ the contribution from the $f_{\omega^{\prime}}^{H^{+}}$modes to $f_{\omega}^{\text {in }}$ is

$$
\left(f_{\omega}^{\text {in }}\right)_{H^{+}}=\frac{e^{-i \omega v}}{\sqrt{4 \pi \omega}}=f_{\omega}^{H^{+}} .
$$

Thus

$$
f_{\omega}^{\text {in }}=f_{\omega}^{H^{+}}+\int_{0}^{\infty} d \omega\left[A_{\omega \omega^{\prime}}^{\mathcal{I}^{+}} f_{\omega^{\prime}}^{\mathcal{I}^{+}}+B_{\omega \omega^{\prime}}^{\mathcal{I}^{+}}\left(f_{\omega^{\prime}}^{\mathcal{I}^{+}}\right)^{*}\right]
$$

with $A_{\omega \omega^{\prime}}^{\mathcal{I}^{+}}$given in (5.30). Using the relation $\Gamma(x)=\frac{\Gamma(1+x)}{x}$ one obtains the form used for the numerical computations

$$
A_{\omega \omega^{\prime}}^{\mathcal{I}^{+}}=-\frac{1}{2 \pi} \sqrt{\frac{\omega}{\omega^{\prime}}}(4 M)^{i 4 M \omega^{\prime}} e^{-i\left(\omega-\omega^{\prime}\right) v_{H}} \frac{\Gamma\left(1-i 4 M \omega^{\prime}\right)}{\left[-i\left(\omega-\omega^{\prime}\right)+\epsilon\right]^{1-i 4 M \omega^{\prime}}} .
$$

Then, using the relations (5.25) one finds

$$
B_{\omega \omega^{\prime}}^{\mathcal{I}^{+}}=\frac{1}{2 \pi} \sqrt{\frac{\omega}{\omega^{\prime}}}(4 M)^{-i 4 M \omega^{\prime}} e^{-i\left(\omega+\omega^{\prime}\right) v_{H}} \frac{\Gamma\left(1+i 4 M \omega^{\prime}\right)}{\left[-i\left(\omega+\omega^{\prime}\right)+\epsilon\right]^{1+i 4 M \omega^{\prime}}} .
$$

In what follows the superscript $\mathcal{I}^{+}$on the matching coefficients $\mathrm{A}$ and $\mathrm{B}$ will be suppressed.

Next, with the aim of finding the components of the stress-energy tensor using (6.5), we construct the Hadamard form of Green's function which in 2D is

$$
G^{(1)}\left(x, x^{\prime}\right)=\int_{0}^{\infty} d \omega\left[f_{\omega}^{\text {in }}(x) f_{\omega}^{\text {in } *}\left(x^{\prime}\right)+f_{\omega}^{\text {in }}\left(x^{\prime}\right) f_{\omega}^{\text {in } *}(x)\right] .
$$

Substituting (6.10) into (6.13) gives

$$
\begin{aligned}
G^{(1)}\left(x, x^{\prime}\right) & =\int_{0}^{\infty} d \omega\left\{\left[f_{\omega}^{H^{+}}(x)+\int_{0}^{\infty} d \omega_{1}\left[A_{\omega \omega_{1}} f_{\omega_{1}}^{\mathcal{I}^{+}}(x)+B_{\omega \omega_{1}} f_{\omega_{1}}^{\mathcal{I}^{+}} *(x)\right]\right]\right. \\
& \times\left[f_{\omega}^{H^{+} *}\left(x^{\prime}\right)+\int_{0}^{\infty} d \omega_{2}\left[A_{\omega \omega_{2}}^{*} f_{\omega_{2}}^{\mathcal{I}^{+} *}\left(x^{\prime}\right)+B_{\omega \omega_{2}}^{*} f_{\omega_{2}}^{\mathcal{I}^{+}}\left(x^{\prime}\right)\right]\right] \\
& +\left[f_{\omega}^{H^{+}}\left(x^{\prime}\right)+\int_{0}^{\infty} d \omega_{1}\left[A_{\omega \omega_{1}} f_{\omega_{1}}^{\mathcal{I}^{+}}\left(x^{\prime}\right)+B_{\omega \omega_{1}} f_{\omega_{1}}^{\mathcal{I}^{+} *}\left(x^{\prime}\right)\right]\right] \\
& \times\left[f_{\omega}^{H^{+} *}(x)+\int_{0}^{\infty} d \omega_{2}\left[A_{\omega \omega_{2}}^{*} f_{\omega_{2}}^{\mathcal{I}^{+} *}(x)+B_{\omega \omega_{2}}^{*} f_{\omega_{2}}^{\mathcal{I}^{+}}(x)\right]\right] .
\end{aligned}
$$

Expanding the integrand of the integral over $\omega$ results in three types of expressions: an integral consisting of products of the modes $f_{\omega}^{H^{+}}$, which we call $G_{A}$, another integral which includes cross products between the modes $f_{\omega}^{H^{+}}$and
$f_{\omega}^{\mathcal{I}^{+}}$, which we call $G_{B}$, and finally an integral consisting of products of the modes $f_{\omega}^{\mathcal{I}^{+}}$, which we called $G_{C}$.

To renormalize we follow a procedure equivalent to that outlined in Sec. VIA. We begin by subtracting off the 
integrals with the integrand evaluated in the large $\omega$ limit. When we add them back, we get contributions that are identical to those obtained for the Unruh state. We are not quite subtracting off the Unruh modes because the large $\omega$ solutions have a dependence on $v_{H}$. However, when the subtracted terms are added back and the integral over $\omega$ is computed, then factors of $\delta\left(\omega_{1}-\omega_{2}\right)$ and $\delta\left(\omega_{1}+\omega_{2}\right)$ are obtained. Note that terms proportional to $\delta\left(\omega_{1}+\omega_{2}\right)$ vanish. For the ones that do not vanish, once one integrates over say $\omega_{2}$, the dependence on $v_{H}$ vanishes.

In Appendix B it is shown that when this method is applied to $G^{(1)}\left(x, x^{\prime}\right)$, the $\Delta G_{A}$ term vanishes. It is also shown that, while the $\Delta G_{B}$ term does not vanish, it does not contribute to the stress-energy tensor. As a result, the only term that contributes to $\Delta\left\langle T_{a b}\right\rangle$ is $\Delta G_{C}\left(x, x^{\prime}\right)$ which has the form

$$
\begin{aligned}
\Delta G_{C}\left(x, x^{\prime}\right)= & \int_{0}^{\infty} d \omega_{1} \int_{0}^{\infty} d \omega_{2}\left\{\left[f_{\omega_{1}}^{\mathcal{I}^{+}}(x) f_{\omega_{2}}^{\mathcal{I}^{+} *}\left(x^{\prime}\right)+f_{\omega_{1}}^{\mathcal{I}^{+}}\left(x^{\prime}\right) f_{\omega_{2}}^{\mathcal{I}^{+} *}(x)\right] \Delta I_{1}\right. \\
& +\left[f_{\omega_{1}}^{\mathcal{I}^{+}}(x) f_{\omega_{2}}^{\mathcal{I}^{+}}\left(x^{\prime}\right)+f_{\omega_{1}}^{\mathcal{I}^{+}}\left(x^{\prime}\right) f_{\omega_{2}}^{\mathcal{I}^{+}}(x)\right] \Delta I_{2} \\
& +\left[f_{\omega_{1}}^{\mathcal{I}^{+} *}(x) f_{\omega_{2}}^{\mathcal{I}^{+} *}\left(x^{\prime}\right)+f_{\omega_{1}}^{\mathcal{I}^{+} *}\left(x^{\prime}\right) f_{\omega_{2}}^{\mathcal{I}^{+} *}(x)\right] \Delta I_{3} \\
& \left.+\left[f_{\omega_{1}}^{\mathcal{I}^{+} *}(x) f_{\omega_{2}}^{\mathcal{I}^{+}}\left(x^{\prime}\right)+f_{\omega_{1}}^{\mathcal{I}^{+} *}\left(x^{\prime}\right) f_{\omega_{2}}^{\mathcal{I}^{+}}(x)\right] \Delta I_{4}\right\},
\end{aligned}
$$

with

$$
\begin{aligned}
& \Delta I_{1}=\int_{0}^{\infty} d \omega\left\{A_{\omega \omega_{1}} A_{\omega \omega_{2}}^{*}-\mathcal{O}\left(A_{\omega \omega_{1}} A_{\omega \omega_{2}}^{*}\right)\right\}, \\
& \Delta I_{2}=\int_{0}^{\infty} d \omega\left\{A_{\omega \omega_{1}} B_{\omega \omega_{2}}^{*}-\mathcal{O}\left(A_{\omega \omega_{1}} B_{\omega \omega_{2}}^{*}\right)\right\}, \\
& \Delta I_{3}=\int_{0}^{\infty} d \omega\left\{B_{\omega \omega_{1}} A_{\omega \omega_{2}}^{*}-\mathcal{O}\left(B_{\omega \omega_{1}} A_{\omega \omega_{2}}^{*}\right)\right\}, \\
& \Delta I_{4}=\int_{0}^{\infty} d \omega\left\{B_{\omega \omega_{1}} B_{\omega \omega_{2}}^{*}-\mathcal{O}\left(B_{\omega \omega_{1}} B_{\omega \omega_{2}}^{*}\right)\right\} .
\end{aligned}
$$

Here $\mathcal{O}$ indicates the asymptotic behavior of the matching coefficients for $\omega \gg \omega_{1,2}$.

The integrals in (6.16) can be computed analytically. Substituting the explicit expression for A from (6.11) into (6.16a) gives

$$
\begin{gathered}
\Delta I_{1}=\frac{1}{4 \pi^{2} \sqrt{\omega_{1} \omega_{2}}}(4 M)^{i 4 M\left(\omega_{1}-\omega_{2}\right)} e^{i v_{H}\left(\omega_{1}-\omega_{2}\right)} \Gamma\left(1-i 4 M \omega_{1}\right) \Gamma\left(1+i 4 M \omega_{2}\right) \Delta K_{1}, \\
\Delta K_{1}=\lim _{\Lambda \rightarrow \infty}(-i)^{i 4 M \omega_{1}}(i)^{-i 4 M \omega_{2}}\left\{\left[\int_{0}^{\Lambda} d \omega \frac{\omega}{\left(\omega-\omega_{1}+i \epsilon_{1}\right)^{1-i 4 M \omega_{1}}\left(\omega-\omega_{2}-i \epsilon_{2}\right)^{1+i 4 M \omega_{2}}}\right.\right. \\
\left.\left.-\int_{1}^{\Lambda} d \omega \omega^{-1+i 4 M\left(\omega_{1}-\omega_{2}\right)}\right]-\int_{0}^{1} d \omega \omega^{-1+i 4 M\left(\omega_{1}-\omega_{2}\right)}\right\} .
\end{gathered}
$$

First we compute the indefinite integrals and evaluate them at the limits. Since $\epsilon_{1}$ and $\epsilon_{2}$ go to $O^{+}$at the end of the calculation, it is acceptable to add terms containing them to the exponents. The first indefinite integral is

$$
\begin{aligned}
\Delta K_{1 a}= & (-i)^{i 4 M \omega_{1}}(i)^{-i 4 M \omega_{2}} \int_{0}^{\Lambda} d \omega \frac{\omega}{\left(\omega-\omega_{1}+i \epsilon_{1}\right)^{1-i 4 M\left(\omega_{1}-i \epsilon_{1}\right)}\left(\omega-\omega_{2}-i \epsilon_{2}\right)^{1+i 4 M\left(\omega_{2}+i \epsilon_{2}\right)}} \\
= & (-i)^{i 4 M \omega_{1}}(i)^{-i 4 M \omega_{2}}\left[-i \frac{\left(\omega-\omega_{1}+i \epsilon_{1}\right)^{i 4 M\left(\omega_{1}-i \epsilon_{1}\right)}\left(\omega-\omega_{2}-i \epsilon_{2}\right)^{-i 4 M\left(\omega_{2}+i \epsilon_{2}\right)}}{4 M\left(\omega_{1}-\omega_{2}-i \epsilon_{1}-i \epsilon_{2}\right)}\right]_{0}^{\Lambda} \\
= & -i(-i)^{i 4 M \omega_{1}}(i)^{-i 4 M \omega_{2}}\left[\frac{\left(\Lambda-\omega_{1}\right)^{i 4 M \omega_{1}}\left(\Lambda-\omega_{2}\right)^{-i 4 M \omega_{2}}}{4 M\left(\omega_{1}-\omega_{2}\right)-i \epsilon_{1}-i \epsilon_{2}}\right. \\
& \left.-\frac{\left(-\omega_{1}\right)^{i 4 M \omega_{1}}\left(-\omega_{2}\right)^{-i 4 M \omega_{2}}}{4 M\left(\omega_{1}-\omega_{2}-i \epsilon_{1}-i \epsilon_{2}\right)}\right] .
\end{aligned}
$$


Note that after evaluating the integral at the limits, $\epsilon_{1}$ and $\epsilon_{2}$ are set to zero in the exponents because they have no effect there. Also, each term is a combination of a principle value and a term proportional to $\delta\left(\omega_{1}-\omega_{2}\right)$, thus

$$
\begin{aligned}
\Delta K_{1 a}= & e^{2 \pi M\left(\omega_{1}+\omega_{2}\right)}\left[-i \frac{\left(\Lambda-\omega_{1}\right)^{i 4 M \omega_{1}}\left(\Lambda-\omega_{2}\right)^{-i 4 M \omega_{2}}}{4 M\left(\omega_{1}-\omega_{2}\right)}+\frac{\pi}{4 M} \delta\left(\omega_{1}-\omega_{2}\right)\right] \\
& +e^{-2 \pi M\left(\omega_{1}+\omega_{2}\right)}\left[i \frac{\omega_{1}^{i 4 M \omega_{1}} \omega_{2}^{-i 4 M \omega_{2}}}{4 M\left(\omega_{1}-\omega_{2}\right)}-\frac{\pi}{4 M} \delta\left(\omega_{1}-\omega_{2}\right)\right]
\end{aligned}
$$

Here we adopt the notation that the principle value of a term such as $\frac{1}{a \pm i \epsilon}$ is written as $\frac{1}{a}$. The second and third integrals in (6.17b) are

$$
\begin{gathered}
\Delta K_{1 b}=-(-i)^{i 4 M \omega_{1}}(i)^{-i 4 M \omega_{2}} \int_{1}^{\Lambda} d \omega \omega^{-1+i 4 M\left(\omega_{1}-\omega_{2}\right)} \\
=i \frac{e^{2 \pi M\left(\omega_{1}+\omega_{2}\right)}}{4 M\left(\omega_{1}-\omega_{2}\right)}\left[\Lambda^{i 4 M\left(\omega_{1}-\omega_{2}\right)}-1\right] \\
\Delta K_{1 c}=-(-i)^{i 4 M \omega_{1}}(i)^{-i 4 M \omega_{2}} \int_{0}^{1} d \omega \omega^{-1+i 4 M\left(\omega_{1}-\omega_{2}\right)} \\
=-e^{2 \pi M\left(\omega_{1}+\omega_{2}\right)} \int_{-\infty}^{0} d z e^{\left[i 4 M\left(\omega_{1}-\omega_{2}\right)+\epsilon\right] z}=-\frac{e^{2 \pi M\left(\omega_{1}+\omega_{2}\right)}}{i 4 M\left(\omega_{1}-\omega_{2}\right)+\epsilon}=\frac{i e^{2 \pi M\left(\omega_{1}+\omega_{2}\right)}}{4 M\left(\omega_{1}-\omega_{2}\right)-i \epsilon} \\
=\frac{i e^{2 \pi M\left(\omega_{1}+\omega_{2}\right)}}{4 M\left(\omega_{1}-\omega_{2}\right)}-e^{2 \pi M\left(\omega_{1}+\omega_{2}\right)} \frac{\pi}{4 M} \delta\left(\omega_{1}-\omega_{2}\right),
\end{gathered}
$$

where in the integral for $\Delta K_{1 c}$ the change of variable $z=\log \omega$ has been made and an integrating factor $\epsilon$ has been inserted. Combining these results, one finds

$$
\begin{aligned}
\Delta K_{1} & =e^{-2 \pi M\left(\omega_{1}+\omega_{2}\right)}\left[i \frac{\omega_{1}^{i 4 M \omega_{1}} \omega_{2}^{-i 4 M \omega_{2}}}{4 M\left(\omega_{1}-\omega_{2}\right)}-\frac{\pi}{4 M} \delta\left(\omega_{1}-\omega_{2}\right)\right] \\
& =i e^{-2 \pi M\left(\omega_{1}+\omega_{2}\right)} \frac{\omega_{1}^{i 4 M \omega_{1}} \omega_{2}^{-i 4 M \omega_{2}}}{4 M\left(\omega_{1}-\omega_{2}-i \epsilon\right)} .
\end{aligned}
$$

Substituting (6.20a) into (6.17a) gives

$$
\begin{aligned}
\Delta I_{1}= & \frac{i}{4 \pi^{2} \sqrt{\omega_{1} \omega_{2}}}(4 M)^{i 4 M\left(\omega_{1}-\omega_{2}\right)} e^{i v_{H}\left(\omega_{1}-\omega_{2}\right)} \Gamma\left(1-i 4 M \omega_{1}\right) \Gamma\left(1+i 4 M \omega_{2}\right) \\
& \times e^{-2 \pi M\left(\omega_{1}+\omega_{2}\right)} \frac{\omega_{1}^{i 4 M \omega_{1}} \omega_{2}^{-i 4 M \omega_{2}}}{4 M\left(\omega_{1}-\omega_{2}-i \epsilon\right)} .
\end{aligned}
$$

Note that this is a finite contribution to $\Delta G_{c}$ because of the factor of $e^{-2 \pi M\left(\omega_{1}+\omega_{2}\right)}$.

Next consider $\Delta I_{4}$ which is the other term with nonvanishing delta functions:

$$
\begin{aligned}
\Delta I_{4} & =\frac{1}{4 \pi^{2} \sqrt{\omega_{1} \omega_{2}}}(4 M)^{-i 4 M\left(\omega_{1}-\omega_{2}\right)} e^{-i v_{H}\left(\omega_{1}-\omega_{2}\right)} \Gamma\left(1+i 4 M \omega_{1}\right) \Gamma\left(1-i 4 M \omega_{2}\right) \Delta K_{4}, \\
\Delta K_{4}= & \lim _{\Lambda \rightarrow \infty}(-i)^{-i 4 M \omega_{1}}(i)^{i 4 M \omega_{2}}\left\{\left[\int_{0}^{\Lambda} d \omega \frac{\omega}{\left(\omega+\omega_{1}+i \epsilon_{1}\right)^{1+i 4 M \omega_{1}}\left(\omega+\omega_{2}-i \epsilon_{2}\right)^{1-i 4 M \omega_{2}}}\right.\right. \\
& \left.\left.-\int_{1}^{\Lambda} d \omega \omega^{-1-i 4 M\left(\omega_{1}-\omega_{2}\right)}\right]-\int_{0}^{1} d \omega \omega^{-1-i 4 M\left(\omega_{1}-\omega_{2}\right)}\right\} .
\end{aligned}
$$

The integrals in $\Delta K_{4}$ can be computed analytically with the result 


$$
\begin{aligned}
\Delta K_{4 a} & =e^{-2 \pi M\left(\omega_{1}+\omega_{2}\right)} \int_{0}^{\Lambda} d \omega \frac{\omega}{\left(\omega+\omega_{1}+i \epsilon_{1}\right)^{1+i 4 M\left(\omega_{1}+i \epsilon_{1}\right)}\left(\omega+\omega_{2}-i \epsilon_{2}\right)^{1-i 4 M\left(\omega_{2}-i \epsilon_{2}\right)}} \\
& =e^{-2 \pi M\left(\omega_{1}+\omega_{2}\right)} \frac{i}{4 M}\left[\frac{\left(\Lambda+\omega_{1}\right)^{-i 4 M \omega_{1}}\left(\Lambda+\omega_{2}\right)^{i 4 M \omega_{2}}}{\omega_{1}-\omega_{2}+i\left(\epsilon_{1}+\epsilon_{2}\right)}-\frac{\omega_{1}^{-i 4 M \omega_{1}} \omega_{2}^{i 4 M \omega_{2}}}{\omega_{1}-\omega_{2}+i\left(\epsilon_{1}+\epsilon_{2}\right)}\right] \\
\Delta K_{4 b} & =e^{-2 \pi M\left(\omega_{1}+\omega_{2}\right)}\left[-\frac{1}{-i 4 M\left(\omega_{1}-\omega_{2}\right)}+\frac{1}{-i 4 M\left(\omega_{1}-\omega_{2}\right)}\right] \\
\Delta K_{4 c} & =-e^{-2 \pi M\left(\omega_{1}+\omega_{2}\right)} \int_{-\infty}^{0} d z e^{\left[-i 4 M\left(\omega_{1}-\omega_{2}\right)+\epsilon\right] z}=-\frac{e^{-2 \pi M\left(\omega_{1}+\omega_{2}\right)}}{-i 4 M\left(\omega_{1}-\omega_{2}\right)+\epsilon} \\
& =-i \frac{e^{-2 \pi M\left(\omega_{1}+\omega_{2}\right)}}{4 M\left(\omega_{1}-\omega_{2}\right)+i \epsilon}=-i \frac{e^{-2 \pi M\left(\omega_{1}+\omega_{2}\right)}}{4 M\left(\omega_{1}-\omega_{2}\right)}-e^{-2 \pi M\left(\omega_{1}+\omega_{2}\right)} \frac{\pi}{4 M} \delta\left(\omega_{1}-\omega_{2}\right) .
\end{aligned}
$$

Both terms in $\Delta K_{4 a}$ can be written in terms of their principle values added to a term proportional to $\delta\left(\omega_{1}-\omega_{2}\right)$. Combining these terms, the following expression for $\Delta K_{4}$ is obtained:

$$
\begin{aligned}
\Delta K_{4} & =e^{-2 \pi M\left(\omega_{1}+\omega_{2}\right)}\left[-i \frac{\omega_{1}^{-i 4 M \omega_{1}} \omega_{2}^{i 4 M \omega_{2}}}{4 M\left(\omega_{1}-\omega_{2}\right)}-\frac{\pi}{4 M} \delta\left(\omega_{1}-\omega_{2}\right)\right] \\
& =-i e^{-2 \pi M\left(\omega_{1}+\omega_{2}\right)} \frac{\omega_{1}^{-i 4 M \omega_{1}} \omega_{2}^{i 4 M \omega_{2}}}{4 M\left(\omega_{1}-\omega_{2}+i \epsilon\right)} .
\end{aligned}
$$

Finally

$$
\Delta I_{4}=-\frac{i}{4 \pi^{2} \sqrt{\omega_{1} \omega_{2}}}(4 M)^{-i 4 M\left(\omega_{1}-\omega_{2}\right)} e^{-i v_{H}\left(\omega_{1}-\omega_{2}\right)} \Gamma\left(1+i 4 M \omega_{1}\right) \Gamma\left(1-i 4 M \omega_{2}\right) e^{-2 \pi M\left(\omega_{1}+\omega_{2}\right)} \frac{\omega_{1}^{-i 4 M \omega_{1}} \omega_{2}^{i 4 M \omega_{2}}}{4 M\left(\omega_{1}-\omega_{2}+i \epsilon\right)} .
$$

Note that if we let $\omega_{1} \leftrightarrow \omega_{2}$ in the expression (6.20b) for $\Delta I_{1}$, then we get $\Delta I_{4}$ in (6.23b). It is also true that if this switch is made in the entire contribution to the two-point function from $\Delta I_{1}$ then that is equal to the contribution from $\Delta I_{4}$. Finally, the total contribution from $\Delta I_{4}$ can be shown to be the complex conjugate of the total contribution from $\Delta I_{1}$. Thus both contributions are real. Next consider $\Delta I_{2}$

$$
\begin{aligned}
\Delta I_{2}= & -\frac{1}{4 \pi^{2} \sqrt{\omega_{1} \omega_{2}}}(4 M)^{i 4 M\left(\omega_{1}+\omega_{2}\right)} e^{i v_{H}\left(\omega_{1}+\omega_{2}\right)} \Gamma\left(1-i 4 M \omega_{1}\right) \Gamma\left(1-i 4 M \omega_{2}\right) \Delta K_{2}, \\
\Delta K_{2}= & \lim _{\Lambda \rightarrow \infty}(-i)^{i 4 M \omega_{1}}(i)^{i 4 M \omega_{2}}\left\{\left[\int_{0}^{\Lambda} d \omega \frac{\omega}{\left(\omega-\omega_{1}+i \epsilon_{1}\right)^{1-i 4 M \omega_{1}}\left(\omega+\omega_{2}-i \epsilon_{2}\right)^{1-i 4 M \omega_{2}}}\right.\right. \\
& \left.\left.-\int_{1}^{\Lambda} d \omega \omega^{-1+i 4 M\left(\omega_{1}+\omega_{2}\right)}\right]-\int_{0}^{1} d \omega \omega^{-1+i 4 M\left(\omega_{1}+\omega_{2}\right)}\right\},
\end{aligned}
$$

where the integrals in $\Delta K_{2}$ can be computed analytically

$$
\begin{aligned}
\Delta K_{2 a} & =(-i)^{i 4 M \omega_{1}}(i)^{i 4 M \omega_{2}} \int_{0}^{\Lambda} d \omega \frac{\omega}{\left(\omega-\omega_{1}+i \epsilon_{1}\right)^{1-i 4 M\left(\omega_{1}-i \epsilon_{1}\right)}\left(\omega+\omega_{2}-i \epsilon_{2}\right)^{1-i 4 M\left(\omega_{2}-i \epsilon_{2}\right)}} \\
& =-\frac{i}{4 M}(-i)^{i 4 M \omega_{1}}(i)^{i 4 M \omega_{2}}\left\{\frac{\left(\Lambda-\omega_{1}\right)^{i 4 M \omega_{1}}\left(\Lambda+\omega_{2}\right)^{i 4 M \omega_{2}}}{\left[\omega_{1}+\omega_{2}-i\left(\epsilon_{1}+\epsilon_{2}\right)\right]}-\frac{\left(-\omega_{1}\right)^{i 4 M \omega_{1}} \omega_{2}^{i 4 M \omega_{2}}}{\left[\omega_{1}+\omega_{2}-i\left(\epsilon_{1}+\epsilon_{2}\right)\right]}\right\} \\
& =-\frac{i}{4 M} e^{2 \pi M\left(\omega_{1}-\omega_{2}\right)} \frac{\left(\Lambda-\omega_{1}\right)^{i 4 M \omega_{1}}\left(\Lambda+\omega_{2}\right)^{i 4 M \omega_{2}}}{\left[\omega_{1}+\omega_{2}-i\left(\epsilon_{1}+\epsilon_{2}\right)\right]}+\frac{i}{4 M} e^{-2 \pi M\left(\omega_{1}+\omega_{2}\right)} \frac{\omega_{1}^{i 4 M \omega_{1}} \omega_{2}^{i 4 M \omega_{2}}}{\left[\omega_{1}+\omega_{2}-i\left(\epsilon_{1}+\epsilon_{2}\right)\right]}, \\
\Delta K_{2 b} & =\frac{i}{4 M} e^{2 \pi M\left(\omega_{1}-\omega_{2}\right)} \frac{\Lambda^{i 4 M\left(\omega_{1}+\omega_{2}\right)}}{\omega_{1}+\omega_{2}}-\frac{i}{4 M} e^{2 \pi M\left(\omega_{1}-\omega_{2}\right)} \frac{1}{\omega_{1}+\omega_{2}}
\end{aligned}
$$




$$
\begin{aligned}
\Delta K_{2 c} & =-e^{2 \pi M\left(\omega_{1}-\omega_{2}\right)} \int_{-\infty}^{0} d z e^{\left[i 4 M\left(\omega_{1}+\omega_{2}\right)+\epsilon\right] z}=-\frac{e^{2 \pi M\left(\omega_{1}-\omega_{2}\right)}}{i 4 M\left(\omega_{1}+\omega_{2}\right)+\epsilon} \\
& =i \frac{e^{2 \pi M\left(\omega_{1}-\omega_{2}\right)}}{4 M\left(\omega_{1}+\omega_{2}\right)-i \epsilon}=i \frac{e^{2 \pi M\left(\omega_{1}-\omega_{2}\right)}}{4 M\left(\omega_{1}+\omega_{2}\right)}-\frac{\pi}{4 M} \delta\left(\omega_{1}+\omega_{2}\right) .
\end{aligned}
$$

Given that $\delta\left(\omega_{1}+\omega_{2}\right)=0$ since the frequencies are all non-negative, one can set $\epsilon_{1}=\epsilon_{2}=0$. Then

$$
\Delta K_{2}=i e^{-2 \pi M\left(\omega_{1}+\omega_{2}\right)} \frac{\omega_{1}^{i 4 M \omega_{1}} \omega_{2}^{i 4 M \omega_{2}}}{4 M\left(\omega_{1}+\omega_{2}\right)},
$$

and

$$
\Delta I_{2}=-\frac{i}{4 \pi^{2} \sqrt{\omega_{1} \omega_{2}}}(4 M)^{i 4 M\left(\omega_{1}+\omega_{2}\right)} e^{i v_{H}\left(\omega_{1}+\omega_{2}\right)} \Gamma\left(1-i 4 M \omega_{1}\right) \Gamma\left(1-i 4 M \omega_{2}\right) e^{-2 \pi M\left(\omega_{1}+\omega_{2}\right)} \frac{\omega_{1}^{i 4 M \omega_{1}} \omega_{2}^{i 4 M \omega_{2}}}{4 M\left(\omega_{1}+\omega_{2}\right)}
$$

Comparing $\Delta I_{2}$ in (6.16b) and $\Delta I_{3}$ in (6.16c), one can immediately see that their contributions to the two-point function, (6.15), are the complex conjugate of each other if one also takes $\omega_{1} \leftrightarrow \omega_{2}$ in the contribution from $\Delta I_{2}$

$$
\begin{aligned}
\Delta I_{3} & =\left(\Delta I_{2}\right)^{*} \\
& =\frac{i}{4 \pi^{2} \sqrt{\omega_{1} \omega_{2}}}(4 M)^{-i 4 M\left(\omega_{1}+\omega_{2}\right)} e^{-i v_{H}\left(\omega_{1}+\omega_{2}\right)} \Gamma\left(1+i 4 M \omega_{1}\right) \Gamma\left(1+i 4 M \omega_{2}\right) e^{-2 \pi M\left(\omega_{1}+\omega_{2}\right)} \frac{\omega_{1}^{-i 4 M \omega_{1}} \omega_{2}^{-i 4 M \omega_{2}}}{4 M\left(\omega_{1}+\omega_{2}\right)}
\end{aligned}
$$

Substituting (6.20b), (6.23b), (6.26b), and (6.27) into (6.15) one finds

$$
\begin{aligned}
\Delta G_{C}\left(x, x^{\prime}\right)= & \Re\left\{\frac{i}{8 \pi^{3}} \int_{0}^{\infty} \frac{d \omega_{1}}{\omega_{1}} \int_{0}^{\infty} \frac{d \omega_{2}}{\omega_{2}} e^{-2 \pi M\left(\omega_{1}+\omega_{2}\right)}\right. \\
& \times\left\{\left[e^{-i \omega_{1} u_{s}+i \omega_{2} u_{s}^{\prime}}+e^{-i \omega_{1} u_{s}^{\prime}+i \omega_{2} u_{s}}\right] \frac{\left(4 M \omega_{1} e^{\frac{v_{H}}{4 M}}\right)^{4 i M \omega_{1}}}{\left(4 M \omega_{2} e^{\frac{v_{H}}{4 M}}\right)^{4 i M \omega_{2}}} \frac{\Gamma\left(1-4 i M \omega_{1}\right) \Gamma\left(1+4 i M \omega_{2}\right)}{4 M\left(\omega_{1}-\omega_{2}-i \epsilon\right)}\right. \\
& -\left[e^{-i \omega_{1} u_{s}-i \omega_{2} u_{s}^{\prime}}+e^{-i \omega_{1} u_{s}^{\prime}-i \omega_{2} u_{s}}\right]\left(4 M \omega_{1} e^{\frac{v_{H}}{4 M}}\right)^{4 i M \omega_{1}}\left(4 M \omega_{2} e^{\frac{v_{H}}{4 M}}\right)^{4 i M \omega_{2}} \\
& \left.\left.\times \frac{\Gamma\left(1-4 i M \omega_{1}\right) \Gamma\left(1-4 i M \omega_{2}\right)}{4 M\left(\omega_{1}+\omega_{2}\right)}\right\}\right\} .
\end{aligned}
$$

There are infrared divergences in this expression. However, it is easy to see that the derivatives in the general formula for the stress-energy tensor (3.1) bring down factors of $\omega_{1}$ and $\omega_{2}$ which remove these infrared divergences. Recalling that $\Delta G_{C}$ is the only contribution to $\left\langle T_{a b}\right\rangle$ from $\Delta G^{(1)}$, it is straightforward to show using (6.5), (C3), (C5), (C6), and (6.28) that

$$
\begin{aligned}
\Delta\left\langle T_{t t}\right\rangle= & -\left(1-\frac{2 M}{r}\right) \lim _{x^{\prime} \rightarrow x} \frac{1}{4}\left(\Delta G_{C ; t^{\prime} ; r}+\Delta G_{C ; t ; r^{\prime}}\right) \\
= & \Re\left\{\frac { i } { 8 \pi ^ { 3 } } \int _ { 0 } ^ { \infty } d \omega _ { 1 } \int _ { 0 } ^ { \infty } d \omega _ { 2 } e ^ { - 2 \pi M ( \omega _ { 1 } + \omega _ { 2 } ) } \left\{e^{i\left(\omega_{2}-\omega_{1}\right) u_{s}} \frac{\left(4 M \omega_{1} e^{\frac{v_{H}}{4 M}}\right)^{4 i M \omega_{1}}}{\left(4 M \omega_{2} e^{\frac{v_{H}}{4 M}}\right)^{4 i M \omega_{2}}} \frac{\Gamma\left(1-4 i M \omega_{1}\right) \Gamma\left(1+4 i M \omega_{2}\right)}{4 M\left(\omega_{1}-\omega_{2}-i \epsilon\right)}\right.\right. \\
& \left.\left.+e^{-i\left(\omega_{2}+\omega_{1}\right) u_{s}}\left(4 M \omega_{1} e^{\frac{v_{H}}{4 M}}\right)^{4 i M \omega_{1}}\left(4 M \omega_{2} e^{\frac{v_{H}}{4 M}}\right)^{4 i M \omega_{2}} \frac{\Gamma\left(1-4 i M \omega_{1}\right) \Gamma\left(1-4 i M \omega_{2}\right)}{4 M\left(\omega_{1}+\omega_{2}\right)}\right\}\right\} .
\end{aligned}
$$

The integral over $\omega_{2}$ of the first term inside the curly bracket can be written in the form

$$
\begin{aligned}
\Delta\left\langle T_{t t}\right\rangle_{1} & =\int_{0}^{\infty} d \omega_{2} \frac{f\left(\omega_{2}\right)}{\omega_{1}-\omega_{2}-i \epsilon}=\int_{0}^{\infty} d \omega_{2}\left[\frac{f\left(\omega_{2}\right)}{\omega_{1}-\omega_{2}}+i \pi \delta\left(\omega_{1}-\omega_{2}\right)\right] \\
& =\lim _{\epsilon \rightarrow 0^{+}}\left[\int_{0}^{\omega_{1}-\epsilon} d \omega_{2} \frac{f\left(\omega_{2}\right)}{\omega_{1}-\omega_{2}}+\int_{\omega_{1}+\epsilon}^{\infty} d \omega_{2} \frac{f\left(\omega_{2}\right)}{\omega_{1}-\omega_{2}}\right]+i \pi f\left(\omega_{1}\right),
\end{aligned}
$$

where the definition of the Cauchy principal value integral has been explicitly used. 
Thus, extracting the explicit form of the $f\left(\omega_{2}\right)$ from (6.29) and substituting it into (6.30) yields

$$
\begin{aligned}
\Delta\left\langle T_{t t}\right\rangle_{1}= & \Re\left\{\frac { i } { 8 \pi ^ { 3 } } \int _ { 0 } ^ { \infty } d \omega _ { 1 } \left[\int_{0}^{\infty} d \omega_{2} e^{-2 \pi M\left(\omega_{1}+\omega_{2}\right)} e^{i\left(\omega_{2}-\omega_{1}\right) u_{s}}\right.\right. \\
& \left.\left.\times \frac{\left(4 M \omega_{1} e^{\frac{v_{H}}{4 M}}\right)^{4 i M \omega_{1}}}{\left(4 M \omega_{2} e^{\frac{v_{H}}{4 M}}\right)^{4 i M \omega_{2}}} \frac{\Gamma\left(1-4 i M \omega_{1}\right) \Gamma\left(1+4 i M \omega_{2}\right)}{4 M\left(\omega_{1}-\omega_{2}\right)}\right]\right\}-\frac{1}{8 \pi^{2}} \int_{0}^{\infty} d \omega_{1} \frac{e^{-4 \pi M \omega_{1}}}{4 M} \\
& \times \Gamma\left(1-4 i M \omega_{1}\right) \Gamma\left(1+4 i M \omega_{1}\right) .
\end{aligned}
$$

The stress-energy tensor for a massless minimally coupled scalar field in the 2D collapsing null shell spacetime has been previously computed analytically using a different method $[20,24]$ and the stress-energy tensor for the Unruh state has also been computed analytically $[20,29]$. For the difference one finds

$$
\begin{aligned}
\Delta\left\langle T_{u u}\right\rangle & =-\frac{1}{24 \pi}\left[\frac{8 M}{\left(u-v_{0}\right)^{3}}+\frac{24 M^{2}}{\left(u-v_{0}\right)^{4}}\right]-\frac{1}{768 \pi M^{2}}, \\
\Delta\left\langle T_{u v}\right\rangle & =\Delta\left\langle T_{v v}\right\rangle=0 \\
\Delta\left\langle T_{t t}\right\rangle & =\Delta\left\langle T_{u u}\right\rangle+2 \Delta\left\langle T_{u v}\right\rangle+\Delta\left\langle T_{v v}\right\rangle \\
& =-\frac{1}{24 \pi}\left[\frac{8 M}{\left(u-v_{0}\right)^{3}}+\frac{24 M^{2}}{\left(u-v_{0}\right)^{4}}\right]-\frac{1}{768 \pi M^{2}} .
\end{aligned}
$$

Both terms in (6.31) have been computed numerically. In the first integral, the numerical computation has been performed by the symmetric removal of the neighborhood with radius $\epsilon$ about the singular points of the integrand, $\omega_{1}=\omega_{2}$. The integral of the second term in (6.30) has been computed using a more straightforward numerical method. Our results for $\Delta\left\langle T_{t t}\right\rangle$ in (6.30) are shown in Fig. 4.

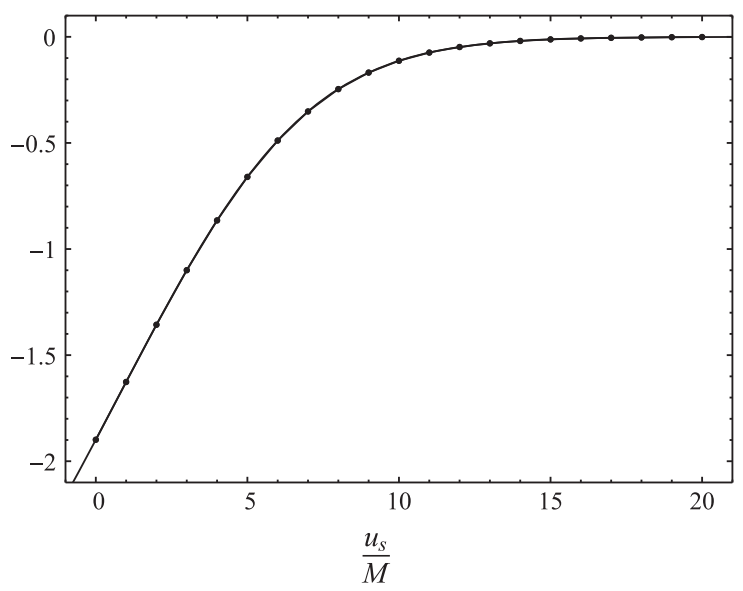

FIG. 4. The quantity $10^{4} M^{2} \Delta\left\langle T_{t t}\right\rangle$ is plotted for the massless minimally coupled scalar field in the region exterior to the null shell and to the event horizon. The dots correspond to the results of the numerical computations. The solid curve represents the analytic results in (6.32).
Although it is not possible to detect this from the plot, our numerical results agree with the analytical results in [20,24] to more than ten digits.

It is worth mentioning that in $2 \mathrm{D}$, once $\Delta\left\langle T_{t t}\right\rangle$ is numerically computed, $\Delta\left\langle T_{r r}\right\rangle$ and $\Delta\left\langle T_{t r}\right\rangle$ can be easily determined from the relations (B7) and (C7).

\section{SUMMARY}

We have presented a method of numerically computing the stress-energy tensor for a massless minimally coupled scalar field in the case when a black hole is formed from the collapse of a spherically symmetric null shell in four dimensions. There are two primary parts to the method. The first is to expand the mode functions in the natural in vacuum state in terms of a complete set of mode functions in the part of Schwarzschild spacetime that is outside of the event horizon of the black hole. Expressions have been found for the matching coefficients that involve integrals of these mode functions over the trajectory of the null shell.

The second part of the method involves subtracting the unrenormalized expression for the stress-energy tensor in the Unruh state from the expression for the unrenormalized stress-energy tensor in the in vacuum state. Since the ultraviolet divergences in the stress-energy tensor are independent of the state, this difference is finite. Then one can add to this the renormalized expression for the stress-energy tensor in the Unruh state that has already been computed $[13,14]$ and the result is the full renormalized stress-energy tensor for the in vacuum state.

We have tested the first part of the method by analytically computing the matching coefficients in the 2D case and reconstructing the mode functions for the in vacuum state. We have also analytically computed the matching coefficients in 4D for the spherically symmetric mode functions (those with $\ell=0$ ) in the in vacuum state for a simple model in which the effective potential in the mode equation is proportional to a Dirac delta function. In this case it was possible to analytically compute the part of the mode function in the in vacuum state that is proportional to $e^{-i \omega v}$ inside the null shell and to verify that it gives the known result on the matching surface. Finally, for the actual case of a collapsing null shell in $4 \mathrm{D}$, we have analytically computed parts of the matching coefficients and used those 
parts to numerically compute part of one of the in modes on the future horizon and shown that it has the correct value at the point where the future horizon intersects the null shell trajectory.

The second part of the method has been tested by numerically computing in 2D the difference between the stress-energy tensor in the in vacuum state for the collapsing null shell spacetime and the Unruh state for Schwarzschild spacetime. The result is in excellent agreement with an analytic expression for the difference obtained from prior calculations of the stress-energy tensor in these two states $[20,24,29]$.

These tests provide substantial evidence that the method will work and that it will be possible to numerically compute the exact renormalized stress-energy tensor for a massless minimally coupled scalar field in a 4D spacetime in which a black hole forms from the collapse of a spherically symmetric null shell. Work on that computation is in progress.

\section{ACKNOWLEDGMENTS}

P. R. A. would like to thank Eric Carlson, Gregory Cook, Charles Evans, Adam Levi, and Amos Ori for helpful conversations and Adam Levi for sharing some of his numerical data. A. F. acknowledges partial financial support from the Spanish Ministerio de Ciencia e Innovación grant FIS2017-84440-C2-1-P and from the Generalitat Valenciana grant PROMETEO/2020/079. This work was supported in part by the National Science Foundation under Grants No. PHY-1308325, No. PHY-1505875, and No. PHY-1912584 to Wake Forest University. Some of the numerical work was done using the WFU DEAC cluster; we thank the WFU Provost's Office and Information Systems Department for their generous support.

\section{APPENDIX A: LAGRANGE INVERSION THEOREM APPLIED TO THE LAMBERT W FUNCTION}

In [33] the relation

$$
e^{-c W(x)}=\sum_{n=0}^{\infty} \frac{c(n+c)^{n-1}}{n !}(-x)^{n}
$$

is derived for any complex constant $c$. An alternative derivation is given here. It is based on the Lagrange inversion theorem [35]. In [36] different forms for the Lagrange inversion theorem are given, one of which we use here. To state the form that is most useful to us we use the notation in [36] that if $f(x)$ is expanded in a Laurent series then $\left[x^{n}\right] f(x)$ denotes the coefficient of $x^{n}$ in that series. Then a statement of the theorem is as follows: Suppose $f$ is a function of $x$ and there is a relation of the following form:

$$
f(x)=x R(f(x)),
$$

where $R(t)$ is a power series in $t$. Suppose further that $\phi(t)$ is also a Laurent series in $t$. Then for any nonzero integer $n$, $\phi(f(x))$ can be expressed in terms of a unique power series in $x$ with coefficients

$$
\left.\left[x^{n}\right] \phi(f) \equiv \frac{1}{n !} \frac{d^{n} \phi(f(x))}{d x^{n}}\right|_{x=0}=\frac{1}{n}\left[t^{n-1}\right] \phi^{\prime}(t) R(t)^{n},
$$

where the interpretation of the far right-hand side is that one first expands the function $\frac{d \phi(t)}{d t} R(t)$ in powers of $t$, then chooses the coefficient of the term proportional to $t^{n-1}$ in that series and divides that coefficient by $n$.

To use this to obtain a power series for the function $e^{-c W(x)}$, note that the Lambert $\mathrm{W}$ function satisfies the relation

$$
W(x)=x e^{-W(x)}=x \sum_{n=0}^{\infty} \frac{(-W(x))^{n}}{n !} .
$$

Thus we can choose the function $R(t)$ in (A2) to be $R(t)=e^{-t}$. We also choose $\phi(t)=e^{-c t}$. Then

$$
\begin{aligned}
{\left[x^{n}\right] e^{-c W(x)} } & =\frac{1}{n}\left[t^{n-1}\right] \phi^{\prime}(t) R^{n}(t) \\
& =-\frac{c}{n}\left[t^{n-1}\right] e^{-(c+n) t}=-\frac{c}{n !}[-(c+n)]^{n-1} \\
& =(-1)^{n} \frac{c}{n !}(c+n)^{n-1} .
\end{aligned}
$$

Equation (A1) follows immediately from this.

\section{APPENDIX B: CONTRIBUTIONS TO THE STRESS-ENERGY TENSOR}

The calculations in this appendix are done entirely for the Schwarzschild geometry. Therefore for simplicity we use $t$ and $u$ to denote the usual time coordinate and the right moving radial null coordinate in Schwarzschild spacetime.

In Sec. VIB it is mentioned that for the null shell spacetime in 2D the Hadamard Green's function in (6.14) can be broken into three parts. One of these, which we call $G_{A}^{(1)}\left(x, x^{\prime}\right)$, includes $f^{H^{+}}$and its complex conjugate and is given by the expression

$G_{A}^{(1)}\left(x, x^{\prime}\right)=\int_{0}^{\infty} d \omega\left\{f_{\omega}^{H^{+}}(x) f_{\omega}^{H^{+} *}\left(x^{\prime}\right)+f_{\omega}^{H^{+}}\left(x^{\prime}\right) f_{\omega}^{H^{+} *}(x)\right\}$.

For the Unruh state the corresponding contribution to $G^{(1)}\left(x, x^{\prime}\right)$ is exactly the same so $\Delta G_{A}^{(1)}\left(x, x^{\prime}\right)=0$.

A second part, $G_{B}^{(1)}\left(x, x^{\prime}\right)$, has terms involving products of $f^{H^{+}}$and its complex conjugate with $f^{\mathcal{I}^{+}}$and its complex conjugate such that 


$$
\begin{aligned}
G_{B}^{(1)}\left(x, x^{\prime}\right)= & \int_{0}^{\infty} d \omega\left\{\int_{0}^{\infty} d \omega_{2}\left[A_{\omega \omega_{2}}^{*} f_{\omega}^{H^{+}}(x) f_{\omega_{2}}^{\mathcal{I}^{+} *}\left(x^{\prime}\right)+B_{\omega \omega_{2}}^{*} f_{\omega}^{H^{+}}(x) f_{\omega_{2}}^{\mathcal{I}^{+}}\left(x^{\prime}\right)\right]\right. \\
& +\int_{0}^{\infty} d \omega_{1}\left[A_{\omega \omega_{1}} f_{\omega}^{H^{+} *}\left(x^{\prime}\right) f_{\omega_{1}}^{\mathcal{I}^{+}}(x)+B_{\omega \omega_{1}} f_{\omega}^{H^{+} *}\left(x^{\prime}\right) f_{\omega_{1}}^{\mathcal{I}^{+}}(x)\right] \\
& +\int_{0}^{\infty} d \omega_{2}\left[A_{\omega \omega_{2}}^{*} f_{\omega}^{H^{+}}\left(x^{\prime}\right) f_{\omega_{2}}^{\mathcal{I}^{+} *}(x)+B_{\omega \omega_{2}}^{*} f_{\omega}^{H^{+}}\left(x^{\prime}\right) f_{\omega_{2}}^{\mathcal{I}^{+}}(x)\right] \\
& \left.+\int_{0}^{\infty} d \omega_{1}\left[A_{\omega \omega_{1}} f_{\omega}^{H^{+} *}(x) f_{\omega_{1}}^{\mathcal{I}^{+}}\left(x^{\prime}\right)+B_{\omega \omega_{1}} f_{\omega}^{H^{+} *}(x) f_{\omega_{1}}^{\mathcal{I}^{+} *}\left(x^{\prime}\right)\right]\right\} .
\end{aligned}
$$

There is no contribution to $G^{(1)}\left(x, x^{\prime}\right)$ which has terms of this form if the field is in the Unruh state, so there is no subtraction term and $\Delta G_{B}^{(1)}\left(x, x^{\prime}\right)=G_{B}^{(1)}\left(x, x^{\prime}\right)$.

While $G_{B}^{(1)}\left(x, x^{\prime}\right)$ contributes to the two-point function, we next show that its contribution to the stress-energy tensor is zero. Substituting (5.20) into (B2) and using (5.23a) and (5.23b), one readily finds that

$$
\begin{aligned}
{\left[G_{B}^{(1)}\left(x, x^{\prime}\right)\right]_{; t ; t^{\prime}}=} & \frac{1}{4 \pi} \int_{0}^{\infty} d \omega\left\{\int_{0}^{\infty} d \omega_{2} \sqrt{\omega \omega_{2}}\left(A_{\omega \omega_{2}}^{*} e^{-i \omega v+i \omega_{2} u^{\prime}}-B_{\omega \omega_{2}}^{*} e^{-i \omega v-i \omega_{2} u^{\prime}}\right)\right. \\
& +\int_{0}^{\infty} d \omega_{1} \sqrt{\omega \omega_{1}}\left(A_{\omega \omega_{1}} e^{i \omega v^{\prime}-i \omega_{1} u}-B_{\omega \omega_{1}} e^{i \omega v^{\prime}+i \omega_{1} u}\right) \\
& +\int_{0}^{\infty} d \omega_{2} \sqrt{\omega \omega_{2}}\left(A_{\omega \omega_{2}}^{*} e^{-i \omega v^{\prime}+i \omega_{2} u}-B_{\omega \omega_{2}}^{*} e^{-i \omega v^{\prime}-i \omega_{2} u}\right) \\
& \left.+\int_{0}^{\infty} d \omega_{1} \sqrt{\omega \omega_{1}}\left(A_{\omega \omega_{1}} e^{i \omega v-i \omega_{1} u^{\prime}}-B_{\omega \omega_{1}} e^{i \omega v+i \omega_{1} u^{\prime}}\right)\right\}, \\
{\left[G_{B}^{(1)}\left(x, x^{\prime}\right)\right]_{; r ; r^{\prime}}=} & \frac{1}{4 \pi\left(1-\frac{2 M}{r}\right)^{2}} \int_{0}^{\infty} d \omega\left\{\int_{0}^{\infty} d \omega_{2} \sqrt{\omega \omega_{2}}\left(-A_{\omega \omega_{2}}^{*} e^{-i \omega v+i \omega_{2} u^{\prime}}+B_{\omega \omega_{2}}^{*} e^{-i \omega v-i \omega_{2} u^{\prime}}\right)\right. \\
& +\int_{0}^{\infty} d \omega_{1} \sqrt{\omega \omega_{1}}\left(-A_{\omega \omega_{1}} e^{i \omega v^{\prime}-i \omega_{1} u}+B_{\omega \omega_{1}} e^{i \omega v^{\prime}+i \omega_{1} u}\right) \\
& +\int_{0}^{\infty} d \omega_{2} \sqrt{\omega \omega_{2}}\left(-A_{\omega \omega_{2}}^{*} e^{-i \omega v^{\prime}+i \omega_{2} u}+B_{\omega \omega_{2}}^{*} e^{-i \omega v^{\prime}-i \omega_{2} u}\right) \\
& \left.+\int_{0}^{\infty} d \omega_{1} \sqrt{\omega \omega_{1}}\left(-A_{\omega \omega_{1}} e^{i \omega v-i \omega_{1} u^{\prime}}+B_{\omega \omega_{1}} e^{i \omega v+i \omega_{1} u^{\prime}}\right)\right\} .
\end{aligned}
$$

From (6.5) one finds

$$
\Delta\left\langle T_{t t}\right\rangle=\frac{1}{4} \lim _{x^{\prime} \rightarrow x}\left[\Delta G_{; t ; t^{\prime}}+\left(1-\frac{2 M}{r}\right)^{2} \Delta G_{; r ; r^{\prime}}\right] .
$$

By substituting (B3) and (B4) into (B5), it is easy to see that the contribution to $\left\langle T_{t t}\right\rangle$ is zero.

Next consider the contribution of $G_{B}^{(1)}\left(x, x^{\prime}\right)$ to $\left\langle T_{r r}\right\rangle$. Using (6.5) it is not hard to show that

$$
\Delta\left\langle T_{r r}\right\rangle=\frac{1}{4} \lim _{x^{\prime} \rightarrow x}\left[\frac{\Delta G_{; t ; t^{\prime}}}{\left(1-\frac{2 M}{r}\right)^{2}}+\Delta G_{; r r^{\prime}}\right]
$$

Together with (B5), one obtains

$$
\Delta\left\langle T_{r r}\right\rangle=\frac{\Delta\left\langle T_{t t}\right\rangle}{\left(1-\frac{2 M}{r}\right)^{2}}
$$

Thus $G_{B}^{(1)}\left(x, x^{\prime}\right)$ does not contribute to $\left\langle T_{r r}\right\rangle$ either.

Finally, we consider the contribution of $G_{B}^{(1)}\left(x, x^{\prime}\right)$ to $\left\langle T_{t r}\right\rangle$. From (6.5) one finds

$$
\Delta\left\langle T_{t r}\right\rangle=\frac{1}{4} \lim _{x^{\prime} \rightarrow x}\left[\Delta G_{; t^{\prime} ; r}+\Delta G_{; t ; r^{\prime}}\right]
$$

Taking the derivative of (B2) with respect to $t$ and $r^{\prime}$, one finds 


$$
\begin{aligned}
{\left[G_{B}^{(1)}\left(x, x^{\prime}\right)\right]_{; t ; r^{\prime}}=} & \frac{1}{4 \pi\left(1-\frac{2 M}{r^{\prime}}\right)} \int_{0}^{\infty} d \omega\left\{\int_{0}^{\infty} d \omega_{2} \sqrt{\omega \omega_{2}}\left(-A_{\omega \omega_{2}}^{*} e^{-i \omega v+i \omega_{2} u^{\prime}}+B_{\omega \omega_{2}}^{*} e^{-i \omega v-i \omega_{2} u^{\prime}}\right)\right. \\
& +\int_{0}^{\infty} d \omega_{1} \sqrt{\omega \omega_{1}}\left(A_{\omega \omega_{1}} e^{i \omega v^{\prime}-i \omega_{1} u}-B_{\omega \omega_{1}} e^{i \omega v^{\prime}+i \omega_{1} u}\right) \\
& +\int_{0}^{\infty} d \omega_{2} \sqrt{\omega \omega_{2}}\left(A_{\omega \omega_{2}}^{*} e^{-i \omega v^{\prime}+i \omega_{2} u}-B_{\omega \omega_{2}}^{*} e^{-i \omega v^{\prime}-i \omega_{2} u}\right) \\
& \left.+\int_{0}^{\infty} d \omega_{1} \sqrt{\omega \omega_{1}}\left(-A_{\omega \omega_{1}} e^{i \omega v-i \omega_{1} u^{\prime}}+B_{\omega \omega_{1}} e^{i \omega v+i \omega_{1} u^{\prime}}\right)\right\}
\end{aligned}
$$

and taking the derivative of (B2) with respect to $t^{\prime}$ and $r$ gives

$$
\begin{aligned}
{\left[G_{B}^{(1)}\left(x, x^{\prime}\right)\right]_{; t^{\prime} ; r}=} & \frac{1}{4 \pi\left(1-\frac{2 M}{r^{\prime}}\right)} \int_{0}^{\infty} d \omega\left\{\int_{0}^{\infty} d \omega_{2} \sqrt{\omega \omega_{2}}\left(A_{\omega \omega_{2}}^{*} e^{-i \omega v+i \omega_{2} u^{\prime}}-B_{\omega \omega_{2}}^{*} e^{-i \omega v-i \omega_{2} u^{\prime}}\right)\right. \\
& +\int_{0}^{\infty} d \omega_{1} \sqrt{\omega \omega_{1}}\left(-A_{\omega \omega_{1}} e^{i \omega v^{\prime}-i \omega_{1} u}+B_{\omega \omega_{1}} e^{i \omega v^{\prime}+i \omega_{1} u}\right) \\
& +\int_{0}^{\infty} d \omega_{2} \sqrt{\omega \omega_{2}}\left(-A_{\omega \omega_{2}}^{*} e^{-i \omega v^{\prime}+i \omega_{2} u}+B_{\omega \omega_{2}}^{*} e^{-i \omega v^{\prime}-i \omega_{2} u}\right) \\
& \left.+\int_{0}^{\infty} d \omega_{1} \sqrt{\omega \omega_{1}}\left(A_{\omega \omega_{1}} e^{i \omega v-i \omega_{1} u^{\prime}}-B_{\omega \omega_{1}} e^{i \omega v+i \omega_{1} u^{\prime}}\right)\right\} .
\end{aligned}
$$

It is clear that $\left[G_{B}^{(1)}\left(x, x^{\prime}\right)\right]_{; t^{\prime} ; r}=-\left[G_{B}^{(1)}\left(x, x^{\prime}\right)\right]_{; t ; r^{\prime}}$ and therefore that their contribution to $\left\langle T_{t r}\right\rangle$ is zero.

The third part of $G^{(1)}\left(x, x^{\prime}\right)$ we call $G_{C}^{(1)}\left(x, x^{\prime}\right)$. Its contribution to $\Delta\left\langle T_{t t}\right\rangle$ is given in Sec. VIB.

\section{APPENDIX C: RELATION BETWEEN TWO COMPONENTS OF $\Delta\left\langle T_{a b}\right\rangle$}

The calculations in this appendix are done entirely for the Schwarzschild geometry. Therefore for simplicity we use $t$ and $u$ to denote the usual time coordinate and the right moving radial null coordinate in Schwarzschild spacetime.

In this appendix a relation is derived between two components of $\Delta\left\langle T_{a b}\right\rangle$ in (6.5) for the 2D collapsing null shell spacetime. As shown in Appendix B only $\Delta G_{C}\left(x, x^{\prime}\right)$ in (6.15) contributes to $\Delta\left\langle T_{a b}\right\rangle$. The explicit form for $\Delta G_{c}\left(x, x^{\prime}\right)$ is

$$
\begin{aligned}
\Delta G_{C}\left(x, x^{\prime}\right)= & \frac{1}{4 \pi} \int_{0}^{\infty} d \omega \int_{0}^{\infty} d \omega_{1} \int_{0}^{\infty} d \omega_{2} \frac{1}{\sqrt{\omega_{1} \omega_{2}}}\left\{\left[A_{\omega \omega_{1}} e^{-i \omega_{1} u}+B_{\omega \omega_{1}} e^{i \omega_{1} u}\right]\right. \\
& \times\left[A_{\omega \omega_{2}}^{*} e^{i \omega_{2} u^{\prime}}+B_{\omega \omega_{2}}^{*} e^{-i \omega_{2} u^{\prime}}\right]+\left[A_{\omega \omega_{1}} e^{-i \omega_{1} u^{\prime}}+B_{\omega \omega_{1}} e^{i \omega_{1} u^{\prime}}\right] \\
& \left.\times\left[A_{\omega \omega_{2}}^{*} e^{i \omega_{2} u}+B_{\omega \omega_{2}}^{*} e^{-i \omega_{2} u}\right]-\text { subtraction terms }\right\},
\end{aligned}
$$

where the subtraction terms have exactly the same form except that the matching coefficients are replaced by the Bogolubov coefficients (5.17) for the Unruh state. Then

$$
\begin{aligned}
{\left[\Delta G_{C}\left(x, x^{\prime}\right)\right]_{; r ; t^{\prime}}=} & \frac{1}{4 \pi} \frac{1}{\left(1-\frac{2 M}{r}\right)} \int_{0}^{\infty} d \omega \int_{0}^{\infty} d \omega_{1} \int_{0}^{\infty} d \omega_{2} \sqrt{\omega_{1} \omega_{2}}\left\{\left[i A_{\omega \omega_{1}} e^{-i \omega_{1} u}-i B_{\omega \omega_{1}} e^{i \omega_{1} u}\right]\right. \\
& \times\left[i A_{\omega \omega_{2}}^{*} e^{i \omega_{2} u^{\prime}}-i B_{\omega \omega_{2}}^{*} e^{-i \omega_{2} u^{\prime}}\right]+\left[-i A_{\omega \omega_{1}} e^{-i \omega_{1} u^{\prime}}+i B_{\omega \omega_{1}} e^{i \omega_{1} u^{\prime}}\right] \\
& \left.\times\left[-i A_{\omega \omega_{2}}^{*} e^{i \omega_{2} u}+i B_{\omega \omega_{2}}^{*} e^{-i \omega_{2} u}\right]-\text { subtraction terms }\right\}
\end{aligned}
$$

A similar calculation for $\left[\Delta G_{C}\left(x, x^{\prime}\right)\right]_{; t ; r^{\prime}}$ gives the opposite sign for each term in square brackets and a replacement of $r$ with $r^{\prime}$ in the overall factor of $\left(1-\frac{2 M}{r}\right)^{-1}$. Thus

$$
\lim _{x^{\prime} \rightarrow x}\left[\Delta G_{C}\left(x, x^{\prime}\right)\right]_{; r ; t^{\prime}}=\lim _{x^{\prime} \rightarrow x}\left[\Delta G_{C}\left(x, x^{\prime}\right)\right]_{; ; ; r^{\prime}}
$$


Next consider

$$
\begin{aligned}
{\left[\Delta G_{C}\left(x, x^{\prime}\right)\right]_{; t ; t^{\prime}}=} & \frac{1}{4 \pi} \int_{0}^{\infty} d \omega \int_{0}^{\infty} d \omega_{1} \int_{0}^{\infty} d \omega_{2} \sqrt{\omega_{1} \omega_{2}}\left\{\left[-i A_{\omega \omega_{1}} e^{-i \omega_{1} u}+i B_{\omega \omega_{1}} e^{i \omega_{1} u}\right]\right. \\
& \times\left[i A_{\omega \omega_{2}}^{*} e^{i \omega_{2} u^{\prime}}-i B_{\omega \omega_{2}}^{*} e^{-i \omega_{2} u^{\prime}}\right]+\left[-i A_{\omega \omega_{1}} e^{-i \omega_{1} u^{\prime}}+i B_{\omega \omega_{1}} e^{i \omega_{1} u^{\prime}}\right] \\
& \left.\times\left[i A_{\omega \omega_{2}}^{*} e^{i \omega_{2} u}-i B_{\omega \omega_{2}}^{*} e^{-i \omega_{2} u}\right]-\text { subtraction terms }\right\}
\end{aligned}
$$

A similar computation for $\left[\Delta G_{C}\left(x, x^{\prime}\right)\right]_{; r ; r^{\prime}}$ gives the relation

$$
\left[\Delta G_{C}\left(x, x^{\prime}\right)\right]_{; r ; r^{\prime}}=\frac{1}{\left(1-\frac{2 M}{r}\right)\left(1-\frac{2 M}{r^{\prime}}\right)}\left[\Delta G_{C}\left(x, x^{\prime}\right)\right]_{; t ; t^{\prime}}
$$

Also a comparison of (C2) and (C4) shows that

$$
\left[\Delta G_{C}\left(x, x^{\prime}\right)\right]_{; r ; t^{\prime}}=-\frac{\left[\Delta G_{C}\left(x, x^{\prime}\right)\right]_{; t ; t^{\prime}}}{1-\frac{2 M}{r}}
$$

Finally by substituting (C5) into (B5) and substituting (C3) and (C6) into (B8) one can see that

$$
\Delta\left\langle T_{r t}\right\rangle=-\frac{\Delta\left\langle T_{t t}\right\rangle}{1-\frac{2 M}{r}}
$$

[1] M. S. Fawcett, Commun. Math. Phys. 89, 103 (1983).

[2] K. W. Howard and P. Candelas, Phys. Rev. Lett. 53, 403 (1984).

[3] K. W. Howard, Phys. Rev. D 30, 2532 (1984).

[4] B. P. Jensen and A. Ottewill, Phys. Rev. D 39, 1130 (1989).

[5] B. P. Jensen, J. G. McLaughlin, and A. C. Ottewill, Phys. Rev. D 43, 4142 (1991).

[6] B. P. Jensen, J. G. Mc Laughlin, and A. C. Ottewill, Phys. Rev. D 45, 3002 (1992).

[7] P. R. Anderson, W. A. Hiscock, and D. A. Samuel, Phys. Rev. Lett. 70, 1739 (1993).

[8] P. R. Anderson, W. A. Hiscock, and D. A. Samuel, Phys. Rev. D 51, 4337 (1995).

[9] P. R. Anderson, W. A. Hiscock, and D. J. Loranz, Phys. Rev. Lett. 74, 4365 (1995).

[10] E. D. Carlson, W. H. Hirsch, B. Obermayer, P. R. Anderson, and P. B. Groves, Phys. Rev. Lett. 91, 051301 (2003).

[11] P. R. Anderson, R. Balbinot, and A. Fabbri, Phys. Rev. Lett. 94, 061301 (2005).

[12] C. Breen and A. C. Ottewill, Phys. Rev. D 85, 084029 (2012).

[13] A. Levi and A. Ori, Phys. Rev. Lett. 117, 231101 (2016).

[14] A. Levi, Phys. Rev. D 95, 025007 (2017).

[15] N. Zilberman, A. Levi, and A. Ori, Phys. Rev. Lett. 124, 171302 (2020).
[16] G. Duffy and A.C. Ottewill, Phys. Rev. D 77, 024007 (2008).

[17] A. Levi, E. Eilon, A. Ori, and M. van de Meent, Phys. Rev. Lett. 118, 141102 (2017).

[18] S. P. Trivedi, Phys. Rev. D 47, 4233 (1993).

[19] G. Vilkovisky, in Quantum Theory of Gravity; Essays in Honor of the 60th Birthday of Bryce S. DeWitt, edited by S. M. Christensen (Adam Hilger Ltd., Bristol, Avon, 1984).

[20] A. Fabbri and J. Navarro-Salas, Modeling Black Hole Evaporation (Imperial College Press, London, United Kingdom, 2005).

[21] S. Massar and R. Parentani, Phys. Rev. D 54, 7444 (1996).

[22] M. R. R. Good, P. R. Anderson, and C. R. Evans, Phys. Rev. D 94, 065010 (2016).

[23] P. R. Anderson, R. D. Clark, A. Fabbri, and M. R. R. Good, Phys. Rev. D 100, 061703(R) (2019).

[24] W. A. Hiscock, Phys. Rev. D 23, 2813 (1981).

[25] S. W. Hawking, Commun. Math. Phys. 43, 199 (1975).

[26] W. G. Unruh, Phys. Rev. D 14, 870 (1976).

[27] T. Elster, Phys. Lett. 94A, 205 (1983).

[28] E. T. Akhmedov, H. Godazgar, and F. K. Popov, Phys. Rev. D 93, 024029 (2016).

[29] P. C. W. Davies, S. A. Fulling, and W. G. Unruh, Phys. Rev. D 13, 2720 (1976).

[30] C. W. Misner, K. S. Thorne, and J. A. Wheeler, Gravitation (Freeman, San Francisco, 1973). 
[31] P. R. Anderson, A. Fabbri, and R. Balbinot, Phys. Rev. D 91, 064061 (2015).

[32] P. R. Anderson, R. Balbinot, A. Fabbri, and R. Parentani, Phys. Rev. D 87, 124018 (2013).

[33] R. M. Corless, D. J. Jeffrey, and D. E. Knuth, in Proceedings of the ACM ISSAC, Maui (Association for Computing Machinery, New York, 1997), p. 135.
[34] S. M. Christensen, Phys. Rev. D 14, 2490 (1976).

[35] C. G. J. Jacobi, J. Reine Angew. Math. 1830, 257 (1826); in Gesammelte Werke, edited by G. Reimer (Berlin, 1891), reprinted by (Chelsea Publishing Company, New York, 1969), Vol. 6, p. 2661.

[36] I. M. Gessel, J. Comb. Theory Ser. A 144, 212 (2016). 